\title{
Over-Sampled Codes Design for PCFM Waveforms based WideBand BeamPattern Synthesis
}

\author{
Ashenafi Yadessa Gemechu
}

\begin{abstract}
The physical emission from a multi-input multioutput(MIMO) radar is generated using multiple-continuous signals called waveforms. In beampattern synthesis, the waveforms are designed to attend a desired emission pattern, i.e. beampattern. In fact, the waveform design in modern radars involve digital signal processing and algorithms implemented on digital computers. That means, the waveform design involves the design of the sampled version or code of the waveform followed by the conversion from the code into the waveform. Based on the Nyquist's criteria, the code needs to be over-sampled to retain more information of the actual waveform. In this paper, the design of over-sampled sequences for the construction of pulse coded frequency modulated(PCFM) waveforms that are used to synthesis a wideband beampattern for radars is dealt. To have good over-sampled, the ratios of out-of-band(OOB) to inband spectral power of the individual sequences are controlled during the design process. In addition, the waveforms, hence, the codes are considered to have constant modulus. The problem is modeled as a non-convex optimization problem. It is solved using the combinations of the alternate directional multiplier $\operatorname{method}(\mathrm{ADMM})$ and majorization-minimization(MM) methods and the Karush-Kuhn-Tucker (KKT) conditions. Simulation results are provided to validate the effectiveness of the proposed method. The simulation results have shown that the method successfully controls the $O O B$ spectral power ratio to force most of the over-sampled sequences fall in the in-band while synthesising wideband beampatterns and satisfying the constant modulus constraint of practical radars.
\end{abstract}

Index Terms-Wideband beampattern, over-sampled, overspecified, in-band, out-of-band, constant modulus, co-existence, spectral power, ADMM, MM, KKT

\section{INTRODUCTION}

$\mathbf{I}$ $\mathrm{N}$ wideband scenario, the transmitter beampattern synthesis involves controlling the radar emission in space and frequency. The process normally focuses on maximizing the power in the target's direction while minimizing the power in the sidelobes. Being specific to the wideband multiinput multi-output(MIMO) radar, the beampattern synthesis is realized through the emission of multiple signals called waveforms from the individual antennas. The waveforms in a physical radar system are basically in continuous form [1], which are designed to meet a goal set for which the emission is generated. On the other hand, the design process employs a digital signal processor(DSP) and algorithms implemented on digital computers. The digital world requires data in discrete form, which mandates the discretization of the waveforms into code sequences. This means the waveform design involves a two-step process, i.e., the code sequence design and the continuous form construction. Here, the code design process

The Author is currently with Addis Ababa Science and Technology University, Addis Ababa, Ethiopia (e-mail: asheyad@gmail.com, ashenafi.y@aastu.edu.et) is governed by the requirements imposed to construct the waveform with enough information. Based on the Nyquist's criteria, the code needs to be over-sampled to retain enough information of the waveform [2], [3].

The waveform design process needs also to consider the limitation of practical transmitters. Transmitting high peak-toaverage(PAPR) waveforms using the High Power Amplifier$\mathrm{s}$ (HPA) operating in saturation cause nonlinear distortion [4]. The nonlinear distortion results in high frequency harmonics, which cause interfering spectral regrowth or spectrum leakage out side of the bandwidth of the radar [5]. In practical system, the out-band leakage of the radar emission is a problem that can interfere and even disrupt the operation of adjacent radiators in radio frequency(RF) spectrum [5], [6]. Such distortion can be minimized by transmitting constant modulus or low PAPR waveforms.

Furthermore, the operation of discrete time fourier transform(DTFT) on over-sampled code sequence produces out-ofband(OOB) power spectrum [3]. Importantly, the design of oversampled sequence to approximate the waveforms cause the distribution of the spectrum power into the outside of the original bandwidth [7]. Unless the OOB power spectrum is minimized so that most of the power spectrum of the corresponding waveform falls in the radars bandwidth, a linear distortion is a result during transmission through the bandlimited transmitter chain of the radar [8]. Such a distortion manifest itself as a form of amplitude ripple formation and phase dispersion. Accompanied with the waveform design, hence, the beampattern synthesis needs to focus in suppressing the OOB leakage or forcing the large portion of the signal energy to fall in the bandwidth of the radar.

Modern radars are normally allocated with large bandwidth, however, they are not usually operate in the whole allocated range [5]. On the other hand, wireless communication is in continuous search for additional bandwidth for its everincreasing services. With such existing problem, the coexistence of communication and radar in the radar band with out affecting the performance of the radar significantly has been proposed as a solution [9]-[11]. In wideband beampattern synthesis, we can even let the two system co-exist in the same frequency range at the same time as long as the direction of the radar main operation, i.e, mainlobe, and that of the communication are different. At this point, the spacefrequency nulling in the emission is a viable solution to realize the co-existence [2] [12] [13].

A large number of researches have been conducted so far to design a wideband beampattern with an underlined assumption that the discrete form is the same as its continuous form, i.e, a waveform. Such an assumption considers the waveforms as 
phase coded signals. The curse of transmitting phase coded signals is that the sudden transition between adjacent chips creates an out-band spectral content that is affected by linear distortions during transmission [14, p. 145-155] [8]. Of course, the works have mainly focused in providing computationally efficient solutions. However, they have missed the important aspect of the problem related to sampling, spectral containment and out-band radiation leakage control.

In [15] the cross-correlation matrix based narrowband method has been extended to design a wideband beampattern using the cross-spectral density matrix(CSDM). Low PAPR signals have been designed to approximate a desired beampattern using CSDM in [18]. In [19] the power spectral density(PSD) has been jointly optimized with a beampattern to approximate a desired beampattern and desired PSD in a mainlobe. The goal of the aforementioned study is to design constant modules signals with good range resolution and orthogonality for multi-target imaging. In [20] [21], the successive closed form (SCF) and a gradiant descent method with projection onto a complex circle manifold methods respectively have been proposed to design a wideband beampattern. The beampattern with interference control (BIC) proposed in [22], solves a wideband beampattern problem while controlling a spectral matching error to realize the co-existence of radar and communication. In [23]-[25], a wideband beampattern has been designed with space-frequency nulling by considering constant modulus signals(codes) while in [26] multiple constraints, i.e., constant modulus, power on each transmitter antenna and total energy have been considered.

The wideband beampattern formulation via Iterative Techniques (WBFIT) method proposed in [17] uses two iterative steps to design a wideband beampattern. In the first step WBFIT iteratively optimizes a matrix of frequency spectrum to approximate a given desired beampattern. Then, it searches for the set of signals whose frequency spectrum best fit with the optimized frequency spectrum iteratively. The work has also provided example on continuous signal(waveform) based wideband beampattern synthesis but it has not dealt with oversampling.

In [2] [12], various practical constraints and problems associated with the practical system have been considered. Paving the road for pratical beampattern design it delineated the line between a waveform and its discrete form. The importance of oversampling and overspecifying a sequence have been discussed deeply. It follows a two-step waveform design, i.e., code design and code-to-waveform conversion. The solutions has also dealt with space-frequency nulling with the emission controlled beampattern using nested loops. In addition, the impact of emission direction on storage of reactive power, which causes damage to the transmitter has been analyzed and the allowable transmission( i.e., for mainlobe ) space has been defined. The method requires the estimation on the number of special spectrum shapes called "beamlets" to define the width of a mainlobe of the beampattern. The work in [27] has considered oversampled code based wideband beampattern synthesis along with frequency spectrum control. Both time oversampling,i.e. by simply increasing the code size , and frequency oversampling have been considered. However, the impact of time oversampling on power spectrum distribution was not properly treated.

This paper focuses on the design of over-sampled sequence to realize pulse coded frequency modulated(PCFM) waveform based wideband beampattern synthesis. It aims to solve the problem with space-frequency nulling having spectrally contained constant modulus waveforms. Specifically, it considers to minimize the OOB spectral power of the individual code sequences, hence, the individual waveforms during the design process. We follow a two step approach as in [2] [12]. First, we design an over-sampled, also over-specified discrete sequence to approximate a desired beampattern with an inequality constraint to impose a space-frequency nulling in a given space and frequency ranges. The OOB power spectrum of each signal is constrained so that most of the signal's power spectrum falls in the considered bandwidth of the signal. Then, the discrete forms are converted into waveforms applying the PCFM method [1] [28].

The contributions of this work are

- the design of spectrally contained over-sampled sequences by controlling the OOB spectral power of each sequence

- the design of an algorithm to solve the problem of beampattern synthesis with space-frequency nulling for wideband radars

- the alternate directional multiplier method(ADMM) and majorization-minimization(MM) methods based iterative solution to tackle the non-convexity of the problem

- Computer simulation based validation to measure the effectiveness of the proposed solution

The rest of the paper is organized as follows. Discussion on a transmit Wideband Beampattern is presented in section II. The effect of oversampling in DTFT-based signal design is analyzed in section III. In section IV, the frequency domain sampling or zero padding is discussed while section $\mathrm{V}$ focuses on the discussion on the importance of controlling the out of band emission and signal spectrum during design. In section $\mathrm{VI}$, the problem formulation is presented and the proposed solution is analyzed in section VII. The convergence of the proposed algorithm is analyzed in section VIII. In section IX, numerical results are provided to validate the proposed solution. At the last, a conclusion is drawn in section X.

Notation: Scalars are denoted by normal lowercase letters, $e$, while vectors and matrices are represented by boldface lowercase, e, and uppercase letters $\mathbf{E}$, respectively. The (.) ${ }^{T}$ and $(.)^{H}$ denote the transpose and Hermitian transpose of a vector or Matrix. $\mathbf{I}_{\widehat{N}}$ represents an $\widehat{N} \times \widehat{N}$ identity matrix. The $|$.$| and \angle$. denote the modulus and phase angle of a complex number respectively. The Euclidean norm is denoted by $\|$.$\| and j=\sqrt{-1}$. Finally, the short forms min and s.t. represent the "minimization" and "subject to" keywords of an optimization problem respectively.

\section{Transmit Wideband Beampattern}

The wideband beampattern design involves the discrete fourier transform(DFT) method, which is applied on signals in their discrete form. The actual radar emission, however, 
is created using continuous signals called waveforms. Bearing this fact in mind, one can visualize the process of the wideband beampattern syntheis,i.e. starting from the code design through the actual emission generation. The discrete form or code is the sampled version of the waveforms. Hence during the code design, we need to take at most care about the approximation of the waveforms from their sampled forms.

Now let us assume a wideband MIMO system of $\mathbf{M}$ transmit uniform linear antennas(ULAs). The radar operates with a central frequency of $f_{c}$ having operational bandwidth of $B$ and time support $T$. Each antenna transmit a constant modulus signal of the form

$$
\mathbf{x}_{m}(t)=\mathbf{s a}_{m}(t) e^{j 2 \pi f_{c}},
$$

where $\mathbf{s a}_{m}(t), m=1 \ldots M$ are the baseband signal given by

$$
\mathbf{s} \mathbf{a}_{m}(t)=\frac{1}{\sqrt{M N}} e^{j \phi_{m}(t)}, m=1, \ldots, \mathbf{M}
$$

where $N$ is the sequence length, $\phi_{m}(t)$ is the phase angles of the $m^{t h}$ signal and $t$ is the time operator.

Ideally, the emission of the radar spread across the frequency range of $\left[-\frac{B}{2}+f_{c}, \frac{B}{2}+f_{c}\right]$ taking $\left[-\frac{B}{2}, \frac{B}{2}\right]$ as an operation bandwidth for its baseband signals.

As per the requirement of DSP, the analog signal $\left\{\mathbf{s a}_{m}(t)\right\}_{m}^{M}$ are sampled into equivalent discrete forms

$$
\mathbf{s}_{m}\left(n t_{s}\right), n=1, \ldots, N, m=1, \ldots, M,
$$

Here, $t_{s}$ is the sampling time given by $t_{s}=\frac{1}{f_{s}}, f_{s}$ is the sampling frequency. To better approximate the waveforms after the sequence is designed, retention of enough information of the waveforms is crucial. The discrete form can retain enough information of the waveforms if it is an over-sampled sequence [7]. If we assume an over-sampling rate of $L \geq 2$, the sampling frequency $f_{s}=L B$ and the sequence length $N=L B T$, where $B T$ is the bandwidth-time product of the radar.

\section{THE EFFECT OF Discrete TIME FOURIER TRANSFORM(DTFT) OF OVER-SAMPLED SEQUENCE ON THE BANDWIDTH OF THE WAVEFORM} by

The DTFT of the discrete signal $\mathbf{s}_{m}, m=1, \ldots, M$ is given

$$
\mathbf{s}_{m}\left(e^{j \omega}\right)=\sum_{n=1}^{N} \mathbf{s}_{m}\left(n t_{s}\right) e^{-j \omega(n-1)}, m=1, \ldots, M
$$

Where the term $e^{-j \omega(n-1)}$ is the DTFT operator and $\omega$ is given in normalized form as $\omega=\frac{2 \pi\left(f-f_{c}\right)}{f_{s}}, f$ is the continuous passband frequency [2]. The DTFT provides a periodic frequency spectrum distribution with period $2 \pi$ making the range of $\omega$ is from $-\pi$ to $\pi$, which corresponds to $\left(f-f_{c}\right) \in\left[\begin{array}{ll}-\frac{f_{s}}{2} & \frac{f_{s}}{2}\end{array}\right]$. Now, we can define the impact of DTFT's operation as follows

- During analysis: the DTFT is based on an existing signal. When the existing signal is sampled according to Nyquist's criteria,i.e., $f_{s} \geq B$, the DTFT repeats the signal spectrum with a period of $2 \pi$ which corresponds to $f_{s}$ having enough separation between the replicas to avoid aliasing.

- During design: an appropriate discrete form is optimized or searched to approximate a desired waveform. The curse is that during the design process the DTFT has the freedom to distribute the power spectrum in the range $\left(f-f_{c}\right) \in\left[\begin{array}{ll}-\frac{f_{s}}{2} & \frac{f_{s}}{2}\end{array}\right]$, which is the baseband frequency. That means, when we have over-sampled sequences during the design process the DTFT makes the power spectrums of the sequences occupy a new bandwidth of $\left[\begin{array}{ll}-\frac{L B}{2} & \frac{L B}{2}\end{array}\right]$ [3], which becomes larger than the bandwidth of the considered waveform or that of the given radar.

So, it is very important to suppress the power spectrum of the designed sequence in the part of the new bandwidth given by $\left[\begin{array}{ll}-\frac{L B}{2} & \frac{B}{2}\end{array}\right] \cup\left[\begin{array}{ll}\frac{B}{2} & \frac{L B}{2}\end{array}\right]$, i.e. OOB, so that most of the power spectrum falls in the original bandwidth. This important action makes the discrete form be a true over-sampled sequence of the waveforms as well as minimizes the OOB power spectrum leakage.

\section{FREQUENCY DOMAIN SAMPLING AND ZERO PADDING}

The DTFT representation is basically in continuous form, which is against the discrete representation requirement of DSPs. The DTFT can be effectively represented by a discrete form,i.e, if its $\left[\begin{array}{ll}-\pi & \pi\end{array}\right]$ normalized-frequency range is sampled at $N$ uniformly spaced frequency points [29, p. 400-401]. As the normalized-frequency range of DTFT corresponds $\left[\begin{array}{ll}-\frac{f_{s}}{2} & \frac{f_{s}}{2}\end{array}\right]$, the resulting DFT spans a frequency range of $f_{s}$ with frequency resolution of $\frac{f_{s}}{N}$. Furthermore, the DFT is required to be over-specified with respect to $N$ to accommodate possible time delays in beamforming [2]. Such overspecification, i.e, frequency domain interpolation, corresponds to zero padding in time domain. The zero padding results in more frequency points, which can make DFT better evaluate the DTFT. One important fact, however, is that zero padding does not provide additional information and does not alter the frequency range of the DFT [29, p. 400] [30, p. 710]. Let $\varpi$ the over-specification rate, then $\mathrm{P}=\varpi N$ is the total number of DFT points. To this end, $P=2 N$, where $N$ is the length of the over-sampled sequence, is sufficient to have a DFT that better samples the DTFT of the over-sampled signal [2]. Now taking the $P=2 N$ frequency points in DFT, the index of the in-band and the OOB ranges in the DFT are defined as $[N-B T+1 \quad N+B T]$ and $\left[\begin{array}{ll}1 & N-B T\end{array}\right] \cup[N+B T+1 \quad P]$ respectively [3].

\section{THE IMPORTANCE OF CONTROLLING THE OUT OF BAND EMISSION AND SIGNAL SPECTRUM DURING DESIGN}

From the previous two section, we know that during DFT based signal design the power spectrum of the waveforms distributed across a new bandwidth, which is greater than the radar bandwidth.

The configuration of an M-antenna ULAs system is shown in fig.1. The signal at far-field at spatial angle $\theta$ for the given 


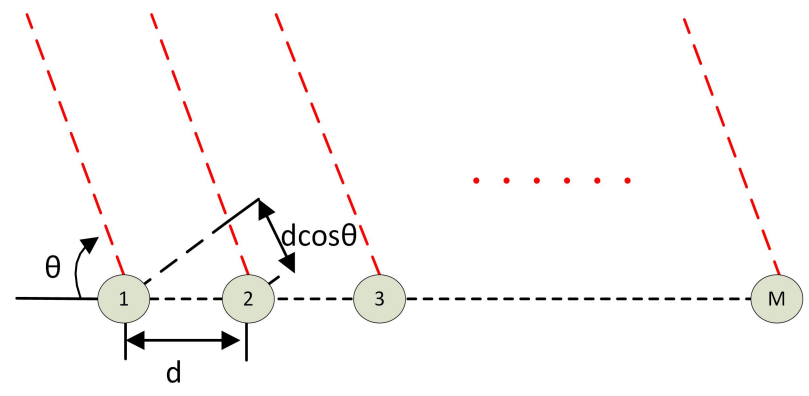

Fig. 1. The configuration of ULAs system with M antennas

configuration is given by

$$
\begin{aligned}
h_{\theta}(t)=\sum_{m=1}^{M} \mathbf{x}_{m}\left(t-\frac{m d \cos \theta}{c}\right) \\
=\sum_{m=1}^{M} \mathbf{s a}_{m}\left(t-\frac{m d \cos \theta}{c}\right) e^{j 2 \pi f_{c}\left(t-\frac{m d \cos \theta}{c}\right)}
\end{aligned}
$$

where $d$ is the inter-elements(inter-antennas) separation and $c$ is the speed of light.

Let $\widehat{f}=f-f_{c}$ and the fourier transform of each of the baseband signals is given by

$$
\widetilde{\mathbf{s}}{ }_{m}(\widehat{f})=\int_{t=0}^{T} \mathbf{s a}_{m}(t) e^{-j 2 \pi \widehat{f} t} d t
$$

where $\widehat{f} \in[-L B / 2 \quad L B / 2]$ as the continuous signal is the result of the the DTFT operation. Defining

$$
U_{\theta}(f)=\sum_{m=1}^{M} \widetilde{\mathbf{s a}}_{m}(\widehat{f}) e^{\frac{-j 2 \pi\left(\hat{f}+f_{c}\right) m d \cos \theta}{c}}
$$

Then the far-field signal

$$
h_{\theta}(t)=\int_{\widehat{f}=-L B / 2}^{L B / 2} U_{\theta}(f) e^{j 2 \pi\left(\widehat{f}+f_{c}\right) t} d \widehat{f}
$$

The relationship in eq.(5) shows that the far-field signal depends on the frequency components of each signal in the range [ $-L B / 2 \quad L B / 2]$, interested readers can refer [2] and [17] for the detail derivation of the far-field signal. And, the beampattern at frequency $f=\widehat{f}+f_{c}$ and spatial angle $\theta$ is defined as $\left|U_{\theta}(f)\right|^{2}=\left|\mathbf{a}^{H}(f, \theta) \widetilde{\mathbf{S a}}(f)\right|^{2}$

where the steering vector

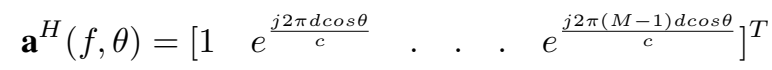

depends on the passband frequency $f$ and

$$
\widetilde{\mathbf{S a}}(\widehat{f})=\left\{\widetilde{\mathbf{s a}}_{m}(\widehat{f})\right\}_{m=1}^{M}
$$

Obviously, beampattern synthesis with waveforms using over-sampled sequence results in an emission with out-ofband(OOB) leakage due to the underlined DTFT operation.

Controlling OOB is mandatory to avoid spurious emissions on adjacent radiators [5]. The controlling is done through the use of solutions that modify the emission at the output of the radar transmitter, for example, using bandpass filters [33] or Predistortion( i.e. the insertion of a nonlinear element prior to the Radio Frequency power amplifier such that the combined transfer characteristic of both is linear which results in baseband interference subtraction of OOB emssion) [34]. The result of such signal modification is that the physical emission is a distorted form of the designed one. So, having a significant portion of the signal power in OOB makes the distortion more severe, which might make the nature of the physical emission deviate from the designed one. In addition, letting the waveforms occupy the new bandwidth might loss the very essence of over-sampling. Hence, the OOB of the designed beampattern as well as that of the individual waveforms need to be minimized so that most of the emmission power falls in the radar bandwidth.

\section{PRoblem Formulation}

Let a continuous spatial angle range $\theta \in\left[\begin{array}{ll}0 & 180^{\circ}\end{array}\right]$ is sampled into $\mathrm{K}$ discrete angle points $\left\{\theta_{k}\right\}_{k=1}^{K}$. The $\left[\begin{array}{ll}-\pi & \pi\end{array}\right]$ bandwidth covered by the DTFT is also sampled into $\mathrm{P}$ discrete DFT points that correspond to $\mathrm{P}$ discrete baseband frequencies

$$
f_{p}=\left(\frac{p}{P}-\frac{1}{2}\right) f_{s}, \quad p=0 \ldots P-1 .
$$

Now, we can define the transmit steering vector of the wideband radar in discrete form as,

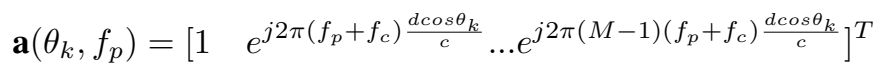

$k=1 \ldots, K, p=1 \ldots P$, where $d$ is inter-element separation of the radar system and given as $d=c / 2\left(f_{c}+B / 2\right)$ to prevent granting lobes in the in-band of the radar [16, section 3.11]. For the sake of clarity we denote $a\left(\theta_{k}, f_{p}\right)$ by $a_{k, p}$ as in [17]. Next, we define the DFT vector using the the DFT points $\frac{f_{p}}{f_{s}}, p=0 \ldots P-1$ as

$$
\mathbf{b}_{p}=\frac{1}{\sqrt{2 N}}\left[\begin{array}{llll}
1 & e^{j 2 \pi v_{p}} & \ldots & e^{j 2 \pi(N-1) v_{p}}
\end{array}\right]^{T}, p=0 \ldots P-1
$$

Where $v_{p}=\left(\frac{p}{P}-\frac{1}{2}\right)$.

Let $\mathbf{F}_{p}=\mathbf{I}_{M} \otimes b_{p}, p=0, \ldots, P-1$. Then, the discrete form of the complex emission of the radar as a function of the passband frequency $f_{p}+f_{c}$ and the discrete azimuthal angle $\theta_{k}$ is given by

$$
\Upsilon_{k, p}=\mathbf{a}_{k, p}^{H} \mathbf{F}_{p} \mathbf{s}, \quad k=1 \ldots K, p=0 \ldots P-1
$$

where $\mathbf{s}=\operatorname{vec}(\mathbf{S}), \mathbf{S}=\left[\mathbf{s}_{1}, \mathbf{s}_{2}, \ldots \mathbf{s}_{M}\right]$. The corresponding power spectrum is given by $\left|\mathbf{a}_{k, p}^{H} \mathbf{F}_{p} \mathbf{s}\right|^{2}$.

Normally, the wideband beampattern can be designed by approximation to a desired beampattern. Here, we approximate the desired beampattern for the mainlobe and sidelobes. Besides to go with the requirement of spectral compatibility, we constraint the spectral space-frequency null power below a predefined limit. Also, the ratio of the OOB power spectrum to the in-band power spectrum is constrained to minimize the power spectrum in the OOB ranges. 
Next we define sets of tuples to facilitate subsequent mathematical formulations as follows.

$$
\bar{\Omega}=\left\{(k n, p n) \mid k n \in\left\{\bar{k}_{1} \ldots \bar{k}_{N u l l}\right\}, p n \in\left\{\bar{p}_{1} \ldots \bar{p}_{N u l l}\right\}\right\}
$$

where $\left\{\bar{k}_{1} \ldots \bar{k}_{N u l l}\right\}$ and $\left\{\bar{p}_{1} \ldots \bar{p}_{N u l l}\right\}$ are the null direction and frequency sets.

$$
\Omega=\{(k, p) \mid k \in\{1 \ldots K\}, p \in\{1 \ldots P\} \wedge(k, p) \notin \bar{\Omega}\}
$$

$\Omega$ is the the set of tuples combining direction and frequency points for the target and sidelobe.

Let $d r_{k, p}$ is the square root of a desired power spectrum at frequency $f_{p}+f_{c}$ and angle $\theta_{k}$ for $(k, p) \in \Omega$.

Then, the given beampattern approximation is defined as

$$
\text { Obj }=\sum_{(k, p) \in \Omega}\left|d r_{k, p}-\xi\right| \mathbf{a}_{k, p}^{H} \mathbf{F}_{p} \mathbf{s}||^{2}
$$

Where $\xi \in \Re$ is a scaling factor respectively.

The total in-band and OOB power spectrum of $\mathbf{s}_{m}$ signal are given as $\sum_{p \in \vartheta_{\text {in }}}\left|\mathbf{b}_{p} \mathbf{s}_{m}\right|^{2}$ and $\sum_{p \in \vartheta_{\text {out }}}\left|\mathbf{b}_{p} \mathbf{s}_{m}\right|^{2}$ respectively, where $\vartheta_{\text {in }}$ and $\vartheta_{\text {out }}$ are the set of in-band and OOB frequency points respectively. The total powers can be reformulated as $\mathbf{s}_{m}^{H} \mathbf{G}_{i n} \mathbf{s}_{m}$ and $\mathbf{s}_{m}^{H} \mathbf{G}_{\text {out }} \mathbf{s}_{m}$, where $\mathbf{G}_{\text {in }}=$ $\sum_{p \in \vartheta_{\text {in }}} \mathbf{b}_{p}^{H} \mathbf{b}_{p}$ and $\mathbf{G}_{\text {out }}=\sum_{p \in \vartheta_{\text {out }}} \mathbf{b}_{p}^{H} \mathbf{b}_{p}$.

Forcing most of the power spectrum to fall in the in-band requires controlling or minimizing the OOB power to inband power ratio. Here, we constraint the ratio to be below a predefined limit, i.e,

$$
\frac{\mathbf{s}_{m}^{H} \mathbf{G}_{\text {out }} \mathbf{s}_{m}}{\mathbf{s}_{m}^{H} \mathbf{G}_{\text {in }} \mathbf{s}_{m}} \leq \Delta, \quad m=1 \ldots M,
$$

where $\Delta$ a maximum $\mathrm{OOB}$ to in-band power spectrum ratio.

In adddition, in wideband scenario the co-existence of radar and communication can be realized using space-frequency nulling. The space-frequency can also be used to mitigate disturbances from an interference source with known frequency and direction. In this work, the space-frequency nulling is enforced by limiting power spectrum in that given frequency and direction to be below certain user defined level. Define the steering vector, the frequency range matrix and angle for nulling as $\widetilde{\mathbf{a}}_{k n, p n}, \widetilde{\mathbf{F}}_{p n}$, and $\widetilde{\theta}_{k n}$,for $(k n, p n) \in \bar{\Omega}$. Then, the nulling enforcement is defined by

$$
\left|\widetilde{\mathbf{a}}_{k n, p n}^{H} \widetilde{\mathbf{F}}_{p n} \mathbf{s}\right|^{2} \leq \frac{\delta}{\xi^{2}}, \quad(k n, p n) \in \bar{\Omega}
$$

where $\delta$ is the user defined null limit, which can be determined based on the interference tolerance of the coexisting radiators in that given space-frequency range and the denominator $\xi \in \Re$ is to account for the same scaling factor with the beampattern approximation.

In accordance with the requirement of HPA, constant modulus waveforms are designed. Hence, the corresponding oversampled sequence can be written as

$$
\mathbf{s}_{m}(n)=\frac{1}{\sqrt{M N}} e^{j \phi_{m}(n)}, n=1 \ldots N, m=1 \ldots \mathbf{M},
$$

Which can be rewritten as

$$
\mathbf{s}=\frac{1}{\sqrt{M N}} e^{j \varphi}
$$

where $\varphi=\left[\phi_{1}^{T} \phi_{2}^{T} \ldots \phi_{M}^{T}\right]^{T}$ is the phase angle of $\mathbf{s}$.

Next, we present the two-phase waveform design process followed in this work.

1) Sequence design: The sampled sequence design of the given problem is formulated in the form of optimization model. To this point, wideband beampattern matching in which the scaled form of a desired beampattern is approximated by the actual one was the preferred approach in most of the previous works.

Having this in mind, the problem is modeled using the following optimization formulation.

$$
\begin{array}{ll} 
& \min _{\mathbf{s}, \varphi} \mathbf{O b j} \\
\text { s.t. } & \left|\widetilde{\mathbf{a}}_{k n, p n}^{H} \widetilde{\mathbf{F}}_{p n} \mathbf{s}\right|^{2} \leq \frac{\delta}{\xi^{2}}, \quad(k n, p n) \in \bar{\Omega} \\
& \frac{\mathbf{s}_{m}^{H} \mathbf{G}_{o u t} \mathbf{s}_{m}}{\mathbf{s}_{m}^{H} \mathbf{G}_{i n} \mathbf{s}_{m}} \leq \Delta, \quad m=1 \ldots M \\
\mathbf{s}= & \frac{1}{\sqrt{M N}} e^{j \varphi}
\end{array}
$$

Where Obj is given by $\mathbf{s}_{m}=\mathbf{s}((m-1) N+1: m N), m=1 \ldots M$.

and

2) Waveform construction: As the actual emission is generated through the transmission of a continuous form or waveform. Hence after appropriate sequences are designed, the sequences need to be converted into the corresponding waveform. In this work, we adopt the construction approach of [1]. First, the designed N-length sequence is converted into a train of $\mathrm{N}$ impulse train with separation $T_{p}$, i.e. the total time of the train is $N T_{p}$. Next, the individual impulses are weighted by coefficients $\alpha_{m n}, m=1 \ldots . M, n=1 \ldots . N$ that account for the phase changes occurring over the corresponding $T_{p}$ intervals and are given by.

$$
\alpha_{m n}= \begin{cases}\bar{\alpha}_{m n} & \text { if }\left|\bar{\alpha}_{m n}\right| \leq \pi \\ \bar{\alpha}_{m n}-2 \pi \operatorname{sgn}\left(\bar{\alpha}_{m n}\right) & \text { Otherwise }\end{cases}
$$

Where

$$
\bar{\alpha}_{m n}=\theta_{m n}-\theta_{m n-1}, m=1 \ldots . M, n=1 \ldots N
$$

$\theta_{m n}$ is the phase value of the $n^{t h}$ chip in the sequence $\mathbf{s}_{m}, m=1 \ldots M . \operatorname{sgn}($.$) is the sign operation.$

Now, collecting the phase changes of the $m^{t h}$ sample into a set

$$
\alpha_{m}=\left[\alpha_{m 1} \alpha_{m 2} \ldots \alpha_{m N}\right]^{T}
$$

and assuming an arbitrary starting phase $\theta_{o}$, the construction of the target PCFM waveform is given by:

$$
\begin{array}{r}
\mathbf{s a}_{m}\left(t: \alpha_{m}\right)= \\
\frac{1}{\sqrt{M N}} e^{\left\{j\left[\int_{0}^{t} g(\tau) *\left[\sum_{n=1}^{N} \alpha_{m n} \sigma\left(\tau-(n-1) T_{p}\right)\right] d \tau+\theta_{o}\right]\right\}}, \\
m=1, \ldots, M . M .
\end{array}
$$

Where,the shaping filter $g(t)$ must integrate to unity in the real line, $\sigma(t)$ is an impulse function and have time support on $\left[0, T_{p}\right]$, * denotes the convolution operation.

For the sake simplicity, we represent $\mathbf{s} \mathbf{a}_{m}\left(t: \alpha_{m}\right)$ as $\mathbf{s} \mathbf{a}_{m}(t)$. 


\section{The Proposed SOLUTION}

In this section, the proposed solution is presented. The objective function in our problem is non-convex. The solution is designed following ADMM and MM methods. First, we turn the non-convex Obj into a more manageable form using the majorization-minimization(MM) method. The conversion has also been used in some of the previous works.

Lemma 1. Applying MM method on Obj and assuming $\boldsymbol{s}^{t}$ as the value of $\mathrm{s}$ at the $t^{\text {th }}$ iteration, the objective function at $\{t+1\}^{\text {th }}$ iteration can be approximated as

$$
\boldsymbol{O b j 1}=\sum_{(k, p) \in \Omega}\left|d r_{k, p} e^{j \angle \boldsymbol{a}_{k, p}^{H} \boldsymbol{F}_{p} \boldsymbol{s}^{t}}-\xi \boldsymbol{a}_{k, p}^{H} \boldsymbol{F}_{p} \boldsymbol{s}\right|^{2}
$$

Proof: see [31]

Proposition 1. let $\boldsymbol{Z}=\left\{z_{k n, p n}\right\}_{k n=1, p n=1}^{K n, P n}$ are auxiliary variables.Then, using lemma 1 the optimization problem can be written as

$$
\begin{array}{ll}
\min _{\boldsymbol{s}, \xi, \boldsymbol{Z}} & \boldsymbol{O b j \mathbf { 1 }} \\
\text { s.t. } & z_{k n, p n}=\xi \widetilde{\boldsymbol{a}}_{k n, p n}^{H} \widetilde{\boldsymbol{F}}_{p n} \boldsymbol{s}, \quad(k n, p n) \in \bar{\Omega} \\
& \left|z_{k n, p n}\right|^{2} \leq \delta, \quad(k n, p n) \in \bar{\Omega} \\
& \frac{\boldsymbol{s}_{m}^{H} \boldsymbol{G}_{\text {out }} \boldsymbol{s}_{m}}{\boldsymbol{s}_{m}^{H} \boldsymbol{G}_{\text {in }} \boldsymbol{s}_{m}} \leq \Delta, \quad m=1 \ldots M \\
\boldsymbol{s}= & \frac{1}{\sqrt{M N}} e^{j \varphi}
\end{array}
$$

Next, we define an augmented lagrangian using the objective function and the linear constraint. Then using the augmented lagrangian and the rest of the constraints we divide the optimization problem in (13) into subproblems, which are more manageable. The solution of the original problem is obtained by orchestrating among the solutions of the subproblems. Let $\rho$ is a penalty factor, also $\mu=\left\{\mu_{k n, p n}\right\}_{k n=1, p n=1}^{K n, P n}$ are dual variables respectively. Then the augmented lagrangian in scaled form [32] with a squared penalty term can be defined as

$$
\begin{aligned}
& \zeta_{\rho}(\mathbf{s}, \xi, \mathbf{Z}, \mu)=\mathbf{O b j} \mathbf{1} \\
+ & \frac{\rho}{2} \sum_{p n=1}^{P n} \sum_{k n=1}^{K n}\left(\left|z_{k n, p n}-\xi \widetilde{\mathbf{a}}_{k n, p n}^{H} \widetilde{\mathbf{F}}_{p n} \mathbf{s}+\frac{\mu_{k n, p n}}{\rho}\right|^{2}-\left|\frac{\mu_{k n, p n}}{\rho}\right|^{2}\right)
\end{aligned}
$$

Proposition 2. [32] The augmented lagrangian along with the rest of the constraints are used to divide the optimization problem into the following subproblems. Accordingly, let $\left\{\boldsymbol{s}^{t}, \xi^{t}, \boldsymbol{Z}^{t}, \mu^{t}\right\}$ are values obtained at $t^{\text {th }}$ step. The values at the $(t+1)^{t h}$ iteration can be obtained using the following subproblems.

$$
\begin{gathered}
\left\{\boldsymbol{s}^{t+1}, \xi^{t+1}\right\}=\underset{\boldsymbol{s}, \xi}{\operatorname{argmin}} \zeta_{\rho}\left(\boldsymbol{s}, \xi^{t}, \boldsymbol{Z}^{t}, \mu^{t}\right) \\
\text { s.t. } \quad \frac{\boldsymbol{s}_{m}^{H} \boldsymbol{G}_{\text {out }} \boldsymbol{s}_{m}}{\boldsymbol{s}_{m}^{H} \boldsymbol{G}_{\text {in }} \boldsymbol{s}_{m}} \leq \Delta, \quad m=1 \ldots M \\
\boldsymbol{s}=\frac{1}{\sqrt{M N}} e^{j \varphi}
\end{gathered}
$$

$$
\begin{aligned}
\left\{z_{k n, p n}^{t+1}\right\}=\underset{z_{k n, p n}}{\operatorname{argmin}} \quad \zeta_{\rho}\left(\boldsymbol{s}^{t+1}, \xi^{t+1}, \boldsymbol{Z}, \mu^{t}\right) \\
\text { s.t. } \quad\left|z_{k n, p n}\right|^{2} \leq \delta, \quad(k n, p n) \in \bar{\Omega} \\
\mu_{k n, p n}^{t+1}=\mu_{k n, p n}^{t}+\rho\left(z_{k n, p n}^{t+1}-\xi^{t+1} \widetilde{\boldsymbol{a}}_{k n, p n}^{H} \widetilde{\boldsymbol{F}}_{p n} \boldsymbol{s}^{t+1}\right), \\
(k n, p n) \in \bar{\Omega}
\end{aligned}
$$

A. Obtaining $\left\{\boldsymbol{s}^{t+1}, \xi^{t+1}\right\}$

Taking the relevant terms from the augmented lagrangian, we can write the following optimization problem

$$
\begin{aligned}
\min _{\mathbf{s}, \xi} & \text { Obj1 } \\
+ & \frac{\rho}{2} \sum_{p n=1}^{P n} \sum_{k n=1}^{K n}\left|z_{k n, p n}^{t}-\xi \widetilde{\mathbf{a}}_{k n, p n}^{H} \widetilde{\mathbf{F}}_{p n} \mathbf{s}+\frac{\mu_{k n, p n}^{t}}{\rho}\right|^{2} \\
\text { s.t. } \quad & \frac{\mathbf{s}_{m}^{H} \mathbf{G}_{o u t} \mathbf{s}_{m}}{\mathbf{s}_{m}^{H} \mathbf{G}_{i n} \mathbf{s}_{m}} \leq \Delta, \quad m=1 \ldots M \\
\mathbf{s} & =\frac{1}{\sqrt{M N}} e^{j \varphi}
\end{aligned}
$$

To simplify the optimization problem we introduce a new auxiliary variable $\overline{\mathbf{s}}=\xi \mathbf{s}$

Lemma 2. Now using the new variable, we can define a new optimization problem as follows

$$
\begin{aligned}
& \min _{\overline{\boldsymbol{s}}, \boldsymbol{s}, \xi} \quad \text { Obj1 } \\
& +\frac{\rho}{2} \sum_{p n=1}^{P n} \sum_{k n=1}^{K n}\left|z_{k n, p n}^{t}-\widetilde{\boldsymbol{a}}_{k n, p n}^{H} \widetilde{\boldsymbol{F}}_{p n} \overline{\boldsymbol{s}}+\frac{\mu_{k n, p n}^{t}}{\rho}\right|^{2} \\
& \text { s.t. } \quad \frac{\overline{\boldsymbol{s}}_{m}^{H} \boldsymbol{G}_{o u t} \overline{\boldsymbol{s}}_{m}}{\overline{\boldsymbol{s}}_{m}^{H} \boldsymbol{G}_{i n} \overline{\boldsymbol{s}}_{m}} \leq \Delta, \quad m=1 \ldots M \\
& \boldsymbol{s}=\frac{1}{\sqrt{M N}} e^{j \varphi}, \quad \overline{\boldsymbol{s}}=\xi \boldsymbol{s}
\end{aligned}
$$

where $\overline{\boldsymbol{s}}_{m}=\overline{\boldsymbol{s}}((m-1) * N+1: m N), m=1 \ldots M$.

Proof: We can show that $\frac{\overline{\boldsymbol{s}}_{m}^{H} \boldsymbol{G}_{\text {out }} \overline{\boldsymbol{s}}_{m}}{\overline{\boldsymbol{s}}_{m}^{H} \boldsymbol{G}_{i n} \overline{\boldsymbol{s}}_{m}}$ is equivalent to $\frac{\boldsymbol{s}_{m}^{H} \boldsymbol{G}_{\text {out }} \boldsymbol{s}_{m}}{\boldsymbol{s}_{m}^{H} \boldsymbol{G}_{\text {in }} \boldsymbol{s}_{m}}$ in optimization terms. Taking the fact that $\xi \in \Re$,
we can have

$$
\frac{\overline{\boldsymbol{s}}_{m}^{H} \boldsymbol{G}_{\text {out }} \overline{\boldsymbol{s}}_{m}}{\overline{\boldsymbol{s}}_{m}^{H} \boldsymbol{G}_{i n} \overline{\boldsymbol{s}}_{m}}=\frac{\xi^{2} \boldsymbol{s}_{m}^{H} \boldsymbol{G}_{\text {out }} \boldsymbol{s}_{m}}{\xi^{2} \boldsymbol{s}_{m}^{H} \boldsymbol{G}_{i n} \boldsymbol{s}_{m}}=\frac{\boldsymbol{s}_{m}^{H} \boldsymbol{G}_{\text {out }} \boldsymbol{s}_{m}}{\boldsymbol{s}_{m}^{H} \boldsymbol{G}_{i n} \boldsymbol{s}_{m}}, m=1 \ldots M
$$

We further define a new augmented lagrangian with a square penalty term.

$$
\begin{array}{r}
\iota_{\gamma}(\overline{\mathbf{s}}, \mathbf{s}, \xi)=\sum_{(k, p) \in \Omega}\left|d r_{k, p} e^{j \angle \mathbf{a}_{k, p}^{H} \mathbf{F}_{p} \mathbf{s}^{t}}-\mathbf{a}_{k, p}^{H} \mathbf{F}_{p} \overline{\mathbf{s}}\right|^{2} \\
+\frac{\rho}{2} \sum_{p n=1}^{P n} \sum_{k n=1}^{K n}\left|z_{k n, p n}^{t}-\widetilde{\mathbf{a}}_{k n, p n}^{H} \widetilde{\mathbf{F}}_{p n} \overline{\mathbf{s}}+\frac{\mu_{k n, p n}^{t}}{\rho}\right|^{2} \\
+\frac{1}{2 \gamma}\|\xi \mathbf{s}-\overline{\mathbf{s}}\|^{2} .
\end{array}
$$


We can see that obtaining $\overline{\mathbf{s}}^{t+1}$ requires solving the following optimization problem

$$
\begin{aligned}
\min _{\overline{\mathbf{s}}} & \iota_{\gamma}\left(\overline{\mathbf{s}}, \mathbf{s}^{t}, \xi^{t}\right) \\
\text { s.t. } & \frac{\overline{\mathbf{s}}_{m}^{H} \mathbf{G}_{\text {out }} \overline{\mathbf{s}}_{m}}{\overline{\mathbf{s}}_{m}^{H} \mathbf{G}_{\text {in }} \overline{\mathbf{s}}_{m}} \leq \Delta, \quad m=1 \ldots M
\end{aligned}
$$

Having $\overline{\mathbf{s}}=\operatorname{vec}\left(\left[\overline{\mathbf{s}}_{1} \overline{\mathbf{s}}_{2} \ldots \overline{\mathbf{s}}_{M}\right]\right)$ in two different forms makes (21) challenging. We circumvent the challenge through an introduction of a new auxiliary variable $\mathbf{y}=\operatorname{vec}\left(\left[\mathbf{y}_{1} \mathbf{y}_{2} \ldots \mathbf{y}_{M}\right]\right)$, where $\xi \mathbf{y}_{m}=\overline{\mathbf{s}}_{m}, m=1 \ldots M \Rightarrow \xi \mathbf{y}=\overline{\mathbf{s}}$.

Hence, we can write

$$
\begin{array}{ll}
\min _{\overline{\mathbf{s}}} & \iota_{\gamma}\left(\overline{\mathbf{s}}, \mathbf{s}^{t}, \xi^{t}\right) \\
\text { s.t. } \quad \xi \mathbf{y}=\overline{\mathbf{s}} & \\
& \quad \frac{\mathbf{y}_{m}^{H} \mathbf{G}_{\text {out }} \mathbf{y}_{m}}{\mathbf{y}_{m}^{H} \mathbf{G}_{\text {in }} \mathbf{y}_{m}} \leq \Delta, \quad m=1 \ldots M
\end{array}
$$

Using a penalty factor $\beta$ we can decouple the two variables as follows

$$
\min _{\overline{\mathbf{s}}} \iota_{\gamma}\left(\overline{\mathbf{s}}, \mathbf{s}^{t}, \xi^{t}\right)+\frac{\beta}{2}\left\|\xi \mathbf{y}^{t}-\overline{\mathbf{s}}\right\|^{2}
$$

And for $m=1 \ldots M$

$$
\begin{array}{cc}
\min _{\mathbf{y}_{m}} & \left\|\xi \mathbf{y}_{m}-\overline{\mathbf{s}}_{m}^{t+1}\right\|^{2} \\
\text { s.t. } & \mathbf{y}_{m}^{H} \mathbf{G} \mathbf{y}_{m} \leq 0
\end{array}
$$

where $\mathbf{G}=\mathbf{G}_{\text {out }}-\Delta \mathbf{G}_{\text {in }}$.

Now, we can obtain $\overline{\mathbf{s}}$ by solving (23) As a result,

$$
\overline{\mathbf{s}}^{t+1}=\mathbf{R}^{-1} \mathbf{r} .
$$

where

$$
\begin{gathered}
\mathbf{R}=2 \sum_{p=1}^{P} \sum_{k=1}^{K} \mathbf{F}_{p}^{H} \mathbf{a}_{k, p} \mathbf{a}_{k, p}^{H} \mathbf{F}_{p}+\left(\frac{1}{\gamma}+\beta\right) \mathbf{I}_{M N} \\
\mathbf{r}=2 \sum_{p=1}^{P} \sum_{k=1}^{K} \mathbf{F}_{p}^{H} \mathbf{a}_{k, p} d r_{k, p} e^{j \angle \mathbf{a}_{k, p}^{H} \mathbf{F}_{p} \mathbf{s}^{t}} \\
+\rho \sum_{p n=1}^{P n} \sum_{k n=1}^{K n} \mathbf{F}_{p n}^{H} \widetilde{\mathbf{a}}_{k n, p n}\left(z_{k n, p n}^{t}+\frac{\mu_{k n, p n}^{t}}{\rho}\right)+\frac{1}{\gamma} \xi^{t} \mathbf{s}^{t}+\beta \xi^{t} \mathbf{y}^{t}
\end{gathered}
$$

To solve the individual $\left\{\mathbf{y}_{m}\right\}_{m=1}^{M}$, we can apply a KKT under projection approach. for $m=1 \ldots M$, let $\overline{\mathbf{y}}_{m}=\overline{\mathbf{s}}_{m}^{t+1} / \xi^{t}$ if $\overline{\mathbf{y}}_{m}^{H} \mathbf{G} \overline{\mathbf{y}}_{m} \leq 0$, then $\mathbf{y}_{m}^{t+1}=\overline{\mathbf{y}}_{m}$. Otherwise solve the following equality constrained optimization problem. And for $m=1 \ldots M$

$$
\begin{array}{cc}
\min _{\mathbf{y}_{m}} & \left\|\mathbf{y}_{m}-\overline{\mathbf{y}}_{m}\right\|^{2} \\
\text { s.t. } & \mathbf{y}_{m}^{H} \mathbf{G} \mathbf{y}_{m}=0
\end{array}
$$

Then, we follow the consensus ADMM approach in [35]. Let $\mathbf{G}=\mathbf{U V} \mathbf{U}^{H}$ is the eigenvalue decomposition with $\mathbf{V}$ is a real matrix holding the eigenvalues of $\mathbf{G}$, and $\mathbf{U}$ is a unitary matrix as $\mathbf{G}$ is hermitian. we formulate the following optimization problem,

$$
\begin{array}{cc}
\min _{y_{m}} & \left\|\widehat{\mathbf{y}}_{m}-\tilde{\mathbf{y}}_{m}\right\|^{2} \\
\text { s.t. } & \widehat{\mathbf{y}}_{m}^{H} \mathbf{V} \widehat{\mathbf{y}}_{m}=0
\end{array}
$$

where $\widehat{\mathbf{y}}_{m}=\mathbf{U}^{H} \mathbf{y}_{m}$ and $\tilde{\mathbf{y}}_{m}=\mathbf{U}^{H} \overline{\mathbf{y}}_{m}$. Define a lagrangian

$$
\chi_{m}\left(\widehat{\mathbf{y}}_{m}, \sigma_{m}\right)=\left\|\widehat{\mathbf{y}}_{m}-\tilde{\mathbf{y}}_{m}\right\|^{2}+\alpha_{m}\left(\widehat{\mathbf{y}}_{m}^{H} \mathbf{V} \widehat{\mathbf{y}}_{m}\right),
$$

where $\alpha_{m}$ is a lagrangian multiplier for the equality constraint associated with $\widehat{\mathbf{y}}_{m}$. Solving

$$
\nabla_{\widehat{\mathbf{y}}_{m}} \chi_{m}\left(\widehat{\mathbf{y}}_{m}, \alpha_{m}\right)=0
$$

results in

$$
\widehat{\mathbf{y}}_{m}=\left(\mathbf{I}_{N}+\alpha_{m} \mathbf{V}\right)^{-1} \tilde{\mathbf{y}}_{m}, m=1 \ldots M
$$

Plugging (28) back to the equality constraint gives as

$$
\Gamma\left(\alpha_{m}\right)=\sum_{n=1}^{N} \frac{V_{n, n}}{\left(1+\alpha_{m} V_{n, n}\right)^{2}}\left|\tilde{\mathbf{y}}_{m}\right|^{2}=0, m=1 \ldots M
$$

which can be solve using the bisection method as in [35],with $\mathbf{I}_{N}+\alpha_{m} \mathbf{V} \succeq 0$ which implies $1+\alpha_{m} V_{n, n} \geq 0$ and $-\frac{1}{v_{\max }} \leq$ $\alpha_{m} \leq-\frac{1}{v_{\text {min }}}$.

Finally,

$$
\mathbf{y}_{m}^{t+1}=\mathbf{U}^{H^{-1}} \widehat{\mathbf{y}}_{m} \quad m=1 \ldots M
$$

And,

$$
\mathbf{y}^{t+1}=\operatorname{vec}\left(\left[\mathbf{y}_{1}^{t+1} \mathbf{y}_{2}^{t+1} \ldots \mathbf{y}_{M}^{t+1}\right]\right)
$$

After obtaining $\overline{\mathbf{s}}^{t+1}$ we can solve for $\mathbf{s}^{t+1}$ and $\xi^{t+1}$.

$$
\begin{array}{r}
\left\{\mathbf{s}^{t+1}\right\}=\underset{\mathbf{s}}{\operatorname{argmin}} \quad\left\|\overline{\mathbf{s}}^{t+1}-\xi^{t} \mathbf{s}\right\|^{2} \\
\text { s.t. } \quad \mathbf{s}=\frac{1}{\sqrt{M N}} e^{j \varphi}
\end{array}
$$

Taking the fact $\xi \in \Re$. The optimal value of $\varphi$ is equal to the phase angle of $\overline{\mathbf{s}}^{t+1}$, i.e., $\varphi^{t+1}=\angle \overline{\mathbf{s}}^{t+1}$. Accordingly, the optimal value of $\mathbf{s}^{t+1}=\frac{1}{\sqrt{M N}} e^{\angle \overline{\mathbf{s}}^{t+1}}$.

Now, we can have the following minimization problem to solve for $\xi^{t+1}$. 


$$
\begin{aligned}
\xi^{t+1}=\underset{\xi}{\operatorname{argmin}} \quad & \frac{1}{2 \gamma}\left\|\xi \frac{1}{\sqrt{M N}} e^{\left\langle\overline{\mathbf{s}}^{t+1}\right.}-\overline{\mathbf{s}}^{t+1}\right\|^{2} \\
& +\frac{\beta}{2}\left\|\xi \overline{\mathbf{y}}^{t+1}-\overline{\mathbf{s}}^{t+1}\right\|^{2} \\
\Rightarrow \xi^{t+1} & =\frac{\sum_{\bar{n}=1}^{M N}\left(\frac{1}{\gamma M N}+\beta\left|y_{\bar{n}}^{t+1}\right|\right)\left|s_{\bar{n}}^{t+1}\right|}{\frac{1}{\gamma}+\beta\left\|\mathbf{y}^{t+1}\right\|^{2}}
\end{aligned}
$$

\section{B. Obtaining $z_{k n, p n}^{t+1}, \quad(k n, p n) \in \bar{\Omega}$}

Once we obtain $\mathbf{s}^{t+1}$ and $\xi^{t+1}$, we formulate optimization problems that can be solved in parallel using only the relevant terms from the augmented lagrangian and the corresponding constraint as follows.

$$
\begin{aligned}
\left\{z_{k n, p n}^{t+1}\right\}=\underset{z_{k n, p n}}{\operatorname{argmin}} \quad \mid z_{k n, p n}-\xi^{t+1} \widetilde{a}_{k n, p n}^{H} \widetilde{F}_{p n} s^{t+1} & \\
& +\left.\frac{\mu_{k n, p n}^{t}}{\rho}\right|^{2} \\
\text { s.t. } & \left|z_{k n, p n}\right|^{2} \leq \delta, \quad(k n, p n) \in \bar{\Omega}
\end{aligned}
$$

Lemma 3. Applying the KKT method, the values of $z_{k n, p n}^{t+1}, \quad(k n, p n) \in \bar{\Omega}$ are obtained as follows

$$
z_{k n, p n}^{t+1}=\frac{\bar{z}_{k n, p n}^{t+1}}{1+\psi_{k n, p n}}
$$

where $\psi_{k n, p n}, \quad(k n, p n) \in \bar{\Omega}$ are lagrangian multipliers given by

$$
\psi_{k n, p n}=\max \left\{0,-1+\frac{\left|\bar{z}_{k n, p n}^{t+1}\right|}{\sqrt{\delta}}\right\}
$$

and

$$
\bar{z}_{k n, p n}^{t+1}=\xi^{t+1} \widetilde{a}_{k n, p n}^{H} \widetilde{F}_{p n} s^{t+1}-\frac{\mu_{k n, p n}^{t}}{\rho}
$$

Proof: see APPENDIX A

Algorithm 1

\section{CONVERGENCE ANALYSis}

Following a proper steps, the convergence of Algorithm 1 is presented in Theorem 1 .

Theorem 1. [36], [37]Taking the fact that the auxiliary variables $z_{k n, p n},(k n, p n) \in \bar{\Omega}$ are bounded and $\rho>0, \beta>0,1 \geq \gamma>0$, if $\left\{\boldsymbol{s}^{t}, \xi^{t},\left\{z_{k n, p n}^{t}\right\}_{(k n, p n) \in \bar{\Omega}},\left\{\mu_{k n, p n}^{t}\right\}_{(k n, p n) \in \bar{\Omega}}\right\} \quad$ and $\left\{\boldsymbol{s}^{t+1}, \xi^{t+1},\left\{z_{k n, p n}^{t+1}\right\}_{(k n, p n) \in \bar{\Omega}},\left\{\mu_{k n, p n}^{t+1}\right\}_{(k n, p n) \in \bar{\Omega}}\right\} \quad$ are sequences generated by Algorithm 1 and assuming $\lim _{t \rightarrow \infty}\left\|\mu^{t+1}-\mu^{t}\right\|^{2} \rightarrow 0$. Then the following inequalities hold

$$
\begin{gathered}
\lim _{t \rightarrow \infty}\left(\zeta_{\rho}\left(\boldsymbol{s}^{t+1}, \xi^{t+1}, \boldsymbol{Z}^{t+1}, \mu^{t}\right)-\zeta_{\rho}\left(\boldsymbol{s}^{t}, \xi^{t}, \boldsymbol{Z}^{t}, \mu^{t}\right)\right) \leq 0 \\
\lim _{t \rightarrow \infty} \zeta_{\rho}\left(\boldsymbol{s}^{t+1}, \xi^{t+1}, \boldsymbol{Z}^{t+1}, \mu^{t+1}\right)-\zeta_{\rho}\left(\boldsymbol{s}^{t+1}, \xi^{t+1}, \boldsymbol{Z}^{t+1}, \mu^{t}\right)=0 . \\
\text { Let } \lim _{t \rightarrow \infty}\left\{\boldsymbol{s}^{t+1}, \xi^{t+1},\left\{z_{k n, p n}^{t+1}\right\}_{(k n, p n) \in \bar{\Omega}},\left\{\mu_{k n, p n}^{t+1}\right\}_{(k n, p n) \in \bar{\Omega}}\right\} \\
\quad \rightarrow\left\{\boldsymbol{s}^{*}, \xi^{*},\left\{z_{k n, p n}^{*}\right\}_{(k n, p n) \in \bar{\Omega}},\left\{\mu_{k n, p n}^{*}\right\}_{(k n, p n) \in \bar{\Omega}}\right\} .
\end{gathered}
$$

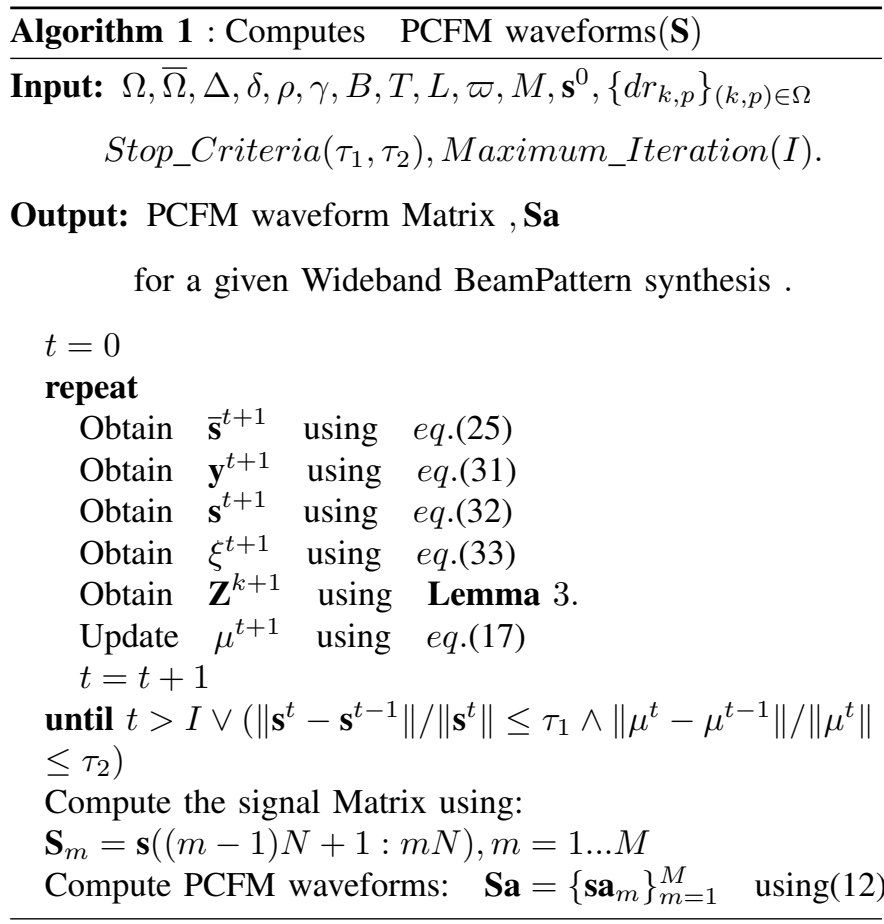

Then, we can show that $0 \in \partial_{s} \zeta_{\rho}(\boldsymbol{s}, \xi, \boldsymbol{Z}, \mu)$ and $\quad 0 \in \partial_{\xi} \zeta_{\rho}(\boldsymbol{s}, \xi, \boldsymbol{Z}, \mu)$. Hence, $\left\{\boldsymbol{s}^{*}, \xi^{*},\left\{z_{k n, p n}^{*}\right\}_{(k n, p n) \in \bar{\Omega}},\left\{\mu_{k n, p n}^{*}\right\}_{(k n, p n) \in \bar{\Omega}}\right\}$ is a limit point and an optimal value.

Proof: See Appendix B.

\section{NumericAl RESUlt}

In this section, the performance of the proposed solution is analyzed using computer simulations. First, results on the convergence property of the solution is presented and analyzed. Following, results to show the impact of oversampling and over-specifying(related to DTFT sampling) on the wideband beampattern are discussed. Finally, discussion on how the performance of the algorithm is influenced by nulling is presented.

The spatial angle range from $0^{\circ}$ to $180^{\circ}$ is discretized to a grid of 181 angle points. A wideband radar operating at a central frequency $f_{c}=1 G H z$ with a bandwidth $B=200 \mathrm{M} \mathrm{Hz}$ is considered. Except for part $B$, the codes are assumed to be twice over-sampled ,i.e. $f_{s}=2 B$ and twice over-specified. In the first part, the bandwidth-time product of $B T=16$ is used, whereas the bandwidth-time product for the following parts is $B T=64$. MATLAB 2016 software and LENOVO Y50P-70 machine with windows 10 are used to conduct the simulations.

\section{A. Convergency Analysis}

Results to show the convergence nature of the proposed solution are presented in fig.2 and fig.3. A 16-antenna wideband MIMO system is considered. The bandwidth-time product, $B T=16$ with twice over-sampling and twice overspecification is used, resulting in a code length of $N=32$ and 64 DFT points. A target is located in $\left[\begin{array}{ll}91^{\circ} & 111^{\circ}\end{array}\right]$ hence 


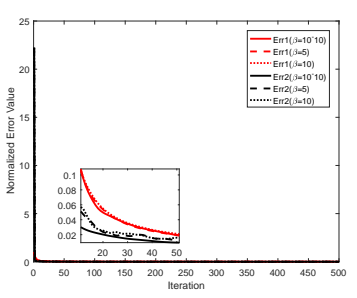

(a) Errors

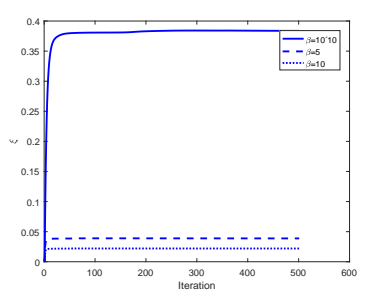

(b) $\xi$
Fig. 2. Errors and $\xi$.

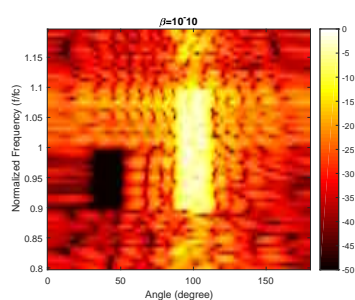

(a) $\beta=10^{-10}$.

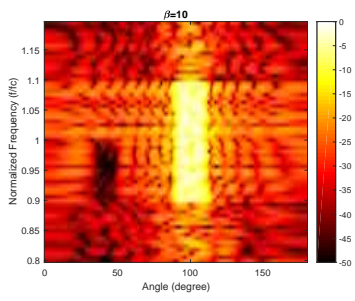

(c) $\beta=10$

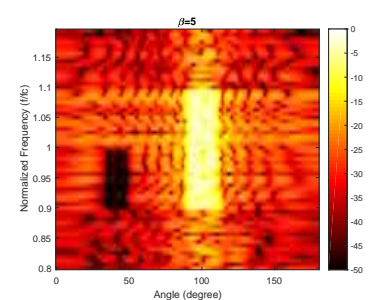

(b) $\beta=5$

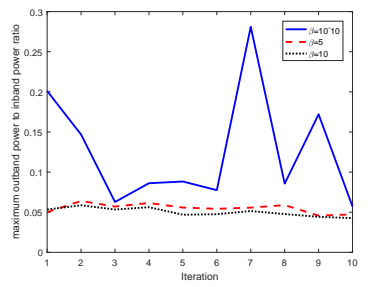

(d) Max.Out_to_InPowerratio
Fig. 3. BeamPatterns and Max outband to inband power ratio.

the beampattern specification is

$$
d r_{k, p}=\left\{\begin{array}{l}
1, \quad p \in \vartheta_{i n}, k \in\left[\begin{array}{ll}
91^{o} & 111^{o}
\end{array}\right] \\
0, \text { Otherwise }
\end{array}\right.
$$

A $-40 d B$ Nulling in the direction of $\left[\begin{array}{ll}35^{\circ} & 50^{\circ}\end{array}\right]$ with frequency range $f_{c}-\frac{B}{2}$ to $f_{c}$, which corresponds to the $[N-B T+1 \quad N]$ DFT range is incorporated.The OOB power to in-band power ratio, $\Delta=6 / 94$, penalty parameters of $\rho=100$ and $\gamma=10^{-3}$ with three values of $\beta=10^{-10}, \beta=5$, and $\beta=10$ are used in the simulations. Two errors are defined as

$\operatorname{Err} 1=\left\|\mu^{t+1}-\mu^{t}\right\| /\left\|\mu^{t+1}\right\| \quad$ and $\quad \operatorname{Err} 2=\left\|s^{t+1}-s^{t}\right\| /\left\|s^{t}\right\|$,

which corresponds to the stopping criteria of the proposed algorithm. For error calculations, 10 runs each with 500 iterations are used. The errors are the average of the 10 runs, whereas the maximum OOB to in-band power ratio is reported per run and the beampatterns are generated by the last run.

The proposed solution combines the beampattern shaping and the OOB to in-band spectral power ratio controlling of the individual signals. Here, the $\beta=10^{-10}$ case represents pure beampattern shaping. The results in fig $2 \mathrm{a}$ show a gradual falling of the errors, which satisfies the condition on the dual variable set for the convergence of the algorithm. When we come to the performance in $\xi$ as depicted in fig. $2 \mathrm{~b}$, all the three cases show stable output. The value of $\xi$ decreases with

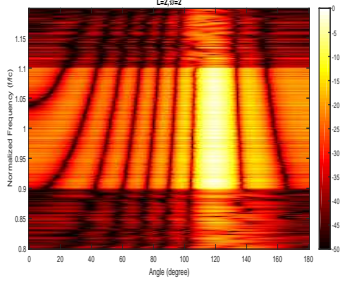

(a) $\mathrm{L}=2, \varpi=2$

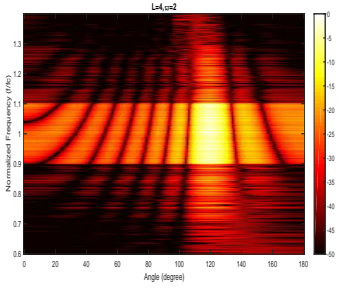

(c) $\mathrm{L}=4, \varpi=2$

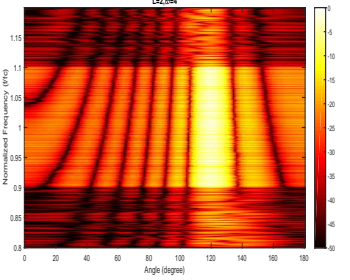

(b) $\mathrm{L}=2, \varpi=4$

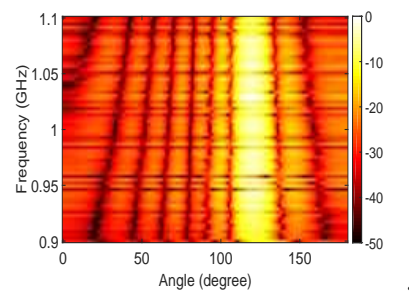

(d) WBFIT
Fig. 4. beampattern

increasing $\beta$. The case with pure beampattern shaping, we can see deep nulling and maximum value for $\xi$. Nevertheless, the maximum $\mathrm{OOB}$ to in-band spectral power ratio of the individual signals, i.e. fig $3 \mathrm{~d}$ shows that the result for pure beampattern fails to satisfy $\Delta$ and show high randomness. This reveals the importance of controlling the individual signals' spectral power along with beampattern shaping when we work with over-sampled sequence. In addition, there is a tradeoff between nulling and the maximum OOB to in-band ratio controlling as shown in fig.3b, fig.3c, fig. $3 \mathrm{~d}$, where a better balance between the two is achieved for $\beta=5$.

\section{B. Effect Oversampling and Overspecifying}

In this part, the performance of the proposed solution is assessed interms of beampattern synthesis, spectral containment and the maximum ratio of OOB to in-band power spectral among the individual waveforms. For comparison WBFIT code length $\mathrm{N}=64$, i.e, a work that represents the previous works, is used. $B T=64, \Delta=4 / 96, \rho=100$ and $\gamma=10^{-3}$ are used. $\beta=10^{-10}$ and $\beta=1$ are also employed. The target direction and number of antennas are $120^{\circ}$ and 10 , which are the same as the values used in WBFIT. In addition, three cases are considered for the proposed solution, i.e. $(L=2, \varpi=2),(L=2, \varpi=4)$, and $(L=4, \varpi=2)$. Note: the WBFIT simulation is using the code provided for the work. A maximum iteration of 1500 , or $\tau_{1}=1 \times 10^{-3}$ and $\tau_{2}=1 \times 10^{-3}$ are the stopping criteria used.

fig. 4 through fig. 7 depict the results of simulations conducted. The beampatterns show from fig.4a-fig. $4 \mathrm{c}$ are that of the proposed solution for the three cases. Compared to the WBFIT beampattern in fig. $4 \mathrm{~d}$, the beampattern show both in-band and OOB emissions with good spectral containment. In all cases, the emission in the target direction is more consistent for the proposed solution than WBFIT. The spectral distribution shown in fig. 5 can be used to make a judgement that the proposed solution show better spectral containment. Here, the 


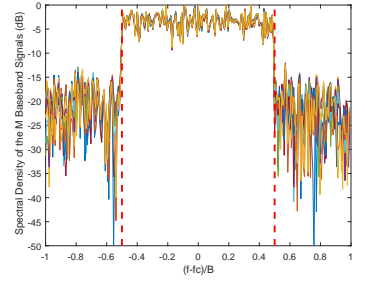

(a) $\mathrm{L}=2, \varpi=2$

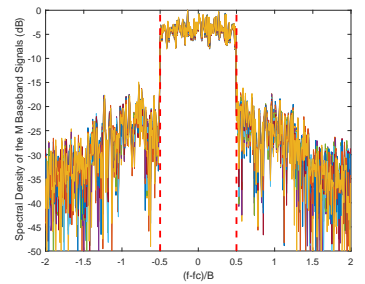

(c) $\mathrm{L}=4, \varpi=2$

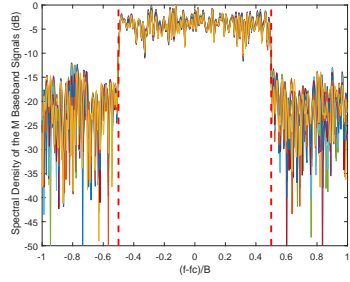

(b) $\mathrm{L}=2, \varpi=4$

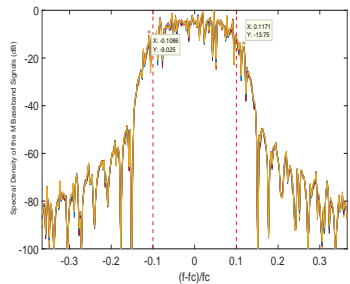

(d) WBFIT
Fig. 5. Overlaid power spectrum of the waveforms

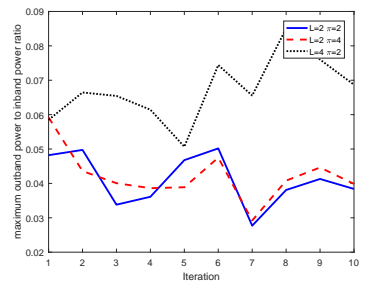

(a) $\beta=10^{-10}$

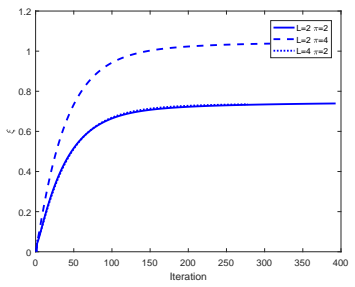

(c) $\beta=10^{-10}$

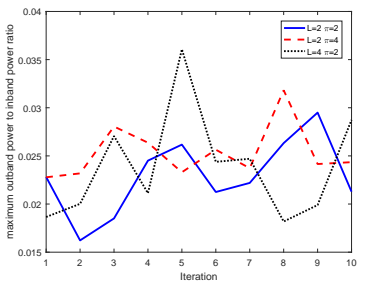

(b) $\beta=1$

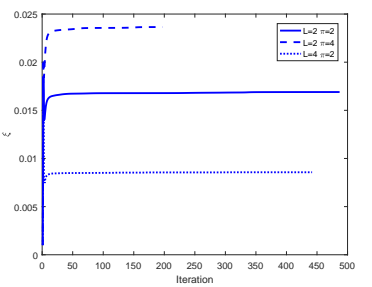

(d) $\beta=1$
Fig. 6. Maximum ratio of out-band to in-band spectral power and $\xi$

result in fig.5d represents the FIR raised cosine filter based waveform formation for WBFIT with over-sampling rate of 5 used after the conversion. Comparing the peak out-band spectral power of the WBFIT to the proposed solution, the WBFIt has the maximum result. In addition, the results show that over-specifying does not alter the spectral distribution of the waveforms. Finally, the maximum ratio of the OOB spectral power ratio and the results of the corresponding $\xi$ 's are shown for two different values of $\beta$ in fig.7. The very small value of $\beta$ is used to minimize the effect of the spectral power control constraint, which is used to emulate the beampattern synthesis without spectral power control for the individual waveforms. According to the results, the proposed solution controls the maximum OOB spectral power of the waveforms using the constraint.

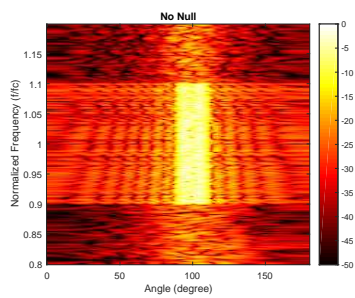

(a) No null

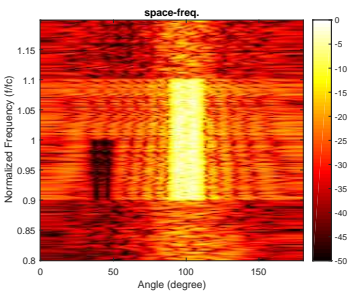

(b) space-freq.

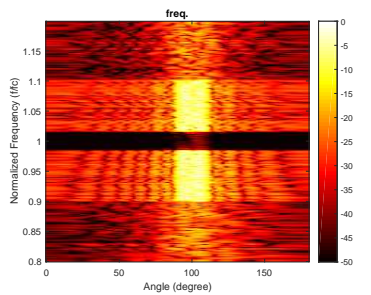

(c) Freq.

Fig. 7. BeamPatterns

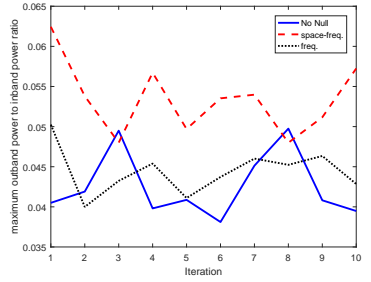

(a) Maximumout-band_to_inbandpowerratio

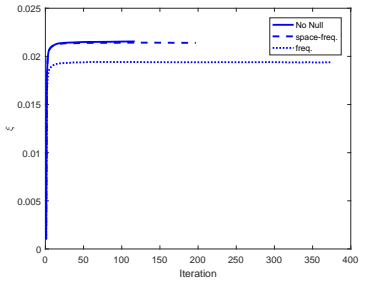

(b) $\xi$
Fig. 8. $\xi$ and maximum out-band to in-band power ratio.

\section{The impact of Nulling on the performance of the algorithm}

Simulation results based on two different scenarios, i.e. without and with nulling, are presented to reflect the impact of nulling on the performance of the proposed solution. In the first scenario a beampattern synthesis without nulling is considered. Whereas, in the second scenario the beampattern synthesis with null is employed. The second scenario consists of two cases. The first case focuses on the problem with spacefrequency nulling in the direction of $\left[\begin{array}{ll}35^{\circ} & 50^{\circ}\end{array}\right]$ with frequency range $f_{c}-\frac{B}{2}$ to $f_{c}$. The second case considers nulling across the frequency region of $\left[\begin{array}{ll}f_{c}-5 \mathrm{MHz} & f_{c}+5 \mathrm{MHz}\end{array}\right]$ in the direction of $\left[0^{\circ} \quad 180^{\circ}\right.$. The nulling depth is $-40 \mathrm{~dB}$. The target direction is $\left[91^{\circ} 111^{\circ}\right]$ hence the beampattern specification is

$$
d r_{k, p}=\left\{\begin{array}{l}
1, p \in \vartheta_{i n}, k \in\left[\begin{array}{ll}
91^{o} & 111^{o}
\end{array}\right] \\
0, \text { Otherwise }
\end{array}\right.
$$

Note: when the nulling space-frequency cell overlaps with the mainlobe, the mainlobe is set to zero for that given spacefrequency cell. $B T=64, L=2, \varpi=2, \Delta=6 / 94, \rho=$ $100, \gamma=10^{-3}$ and $\beta=5$ are the parameters set. A stopping criteria of $\tau_{1}=4 \times 10^{-3}$ and $\tau_{2}=4 \times 10^{-3}$ with a maximum iteration of 600 is used. Ten runs are employed to report the maximum OOB to in-band spectral power ratio, whereas the beampatterns are generated based on the result of the last run. 
The beampattern in fig.7a,fig.7b and fig.7c show significant reduction of the emission power in OOB. The nulling performance for both cases satisfies the limit. Specifically, the nulling in fig.7c shows that the method can support deep nulling when the frequency region of the nulling does not coincide with that of the mainlobe. The value of $\xi$ presented in fig.8a indicates early convergence for all cases for the given error threshold. However, the magnitude of $\xi$ for the third case is less than those of the other two. Regarding the maximum OOB to in-band spectral power ratio, the algorithm satisfies the limit for the three cases. Here when the nulling frequency region overlaps with that of the mainlobe, there is significant increases in the ratio. Hence, space-frequency increases of the maximum OOB to in-band ratio.

\section{Conclusion}

An over-sampling sequence design plays a crucial role in constructing waveforms for wideband beampattern synthesis. In this work, over-sampled sequences to construct PCFM waveforms for a DFT based wideband beampattern synthesis is dealt. The problem focuses on controlling the outband spectral power of the individual sequences so that the designed sequences become good over-sampled version of the considered waveforms. The problem is modeled a nonconvex optimization problem. By applying the ADMM, MM, and KKT methods we tackle the problem systematically. The validation of the proposed solution is made using computer simulations. The results of the simulations show that the method successfully controls the out-band spectral power ratio to force most of the over-sampled sequences fall in the in-band while synthesising wideband beampatterns and satisfying the constant modulus constraint of practical radars. Therefore, the method can be used to design an over-sampled sequence for PCFM waveforms based wideband beampattern sysnthesis.

\section{APPENDix A \\ ProOF OF LEMMA3}

Proof: for $(k n, p n) \in \bar{\Omega}$ Let

$$
\bar{z}_{k n, p n}^{t+1}=\xi^{t+1} \widetilde{a}_{k n, p n}^{H} \widetilde{F}_{p n} s^{t+1}-\frac{\mu_{k n, p n}^{t}}{\rho}
$$

Using the lagrangian inequality multipliers $\psi_{k n, p n}, \quad(k n, p n) \in \bar{\Omega}$, we can define the lagrangian for the individual $z_{k n, p n}$ as

$$
\begin{aligned}
\ell_{k n, p n}\left(z_{k n, p n}, \psi_{k n, p n}\right)= & \left|z_{k n, p n}-\bar{z}_{k n, p n}^{t+1}\right|^{2} \\
& +\psi_{k n, p n}\left(\left|z_{k n, p n}\right|^{2}-\delta\right)
\end{aligned}
$$

Next, we define the KKT for the individual $z_{k n, p n}$ variables

$$
\begin{array}{ll}
\text { 1. } & \left|z_{k n, p n}\right|^{2} \leq \delta \\
\text { 2. } & \psi_{k n, p n} \geq 0 \\
\text { 3. } & \nabla_{z_{k n, p n}} \ell_{k n, p n}\left(z_{k n, p n}, \psi_{k n, p n}\right)=0 \\
\text { 4. } & \psi_{k n, p n}\left(\left|z_{k n, p n}\right|^{2}-\delta\right)=0
\end{array}
$$

Here applying the KKT condition 3 and 2, we can write

$$
z_{k n, p n}^{t+1}=\frac{\bar{z}_{k n, p n}^{t+1}}{1+\psi_{k n, p n}}
$$

Next we solve for $\psi_{k n, p n}$ by plugging (40) into the inequality to satisfy the KKT condition 1 .

$$
\left|\frac{\bar{z}_{k n, p n}^{t+1}}{1+\psi_{k n, p n}}\right|^{2} \leq \delta
$$

which results in the following quadratic inequality.

$$
\left|\psi_{k n, p n}\right|^{2}+2 \psi_{k n, p n}+1-\frac{\left|\bar{z}_{k n, p n}^{t+1}\right|}{\sqrt{\delta}} \geq 0
$$

The inequality in (41) has the following solutions $\psi_{k n, p n} \leq$ $-1-\frac{\left|\bar{z}_{k n, p n}^{t+1}\right|}{\sqrt{\delta}}$ or $\psi_{k n, p n} \geq-1+\frac{\left|\bar{z}_{k n, p}^{t+1}\right|}{\sqrt{\delta}}$. KKT condition 2 . implies $\psi_{k n, p n} \leq-1-\frac{\left|\bar{z}_{k n, p p}^{t+1}\right|}{\sqrt{\delta}}$ cannot be a solution as it is always negative. Taking $\psi_{k n, p n} \geq-1+\frac{\left|\bar{z}_{k n, p n}^{t+1}\right|}{\sqrt{\delta}}$ and KKT condition $2, \psi_{k n, p n} \geq-1+\frac{\left|\bar{z}_{k n, p n}^{t+1}\right|}{\sqrt{\delta}}$ when $\left|\bar{z}_{k n, p n}^{t+1}\right|^{2} \geq \delta$ or $\psi_{k n, p n} \geq 0$ otherwise.

Now, plugging (40) into $\left|z_{k n, p n}-\bar{z}_{k n, p n}^{t+1}\right|^{2}$ gives as the following relationship.

$$
\begin{gathered}
\lim _{\psi_{k n, p n} \rightarrow 0}\left|z_{k n, p n}-\bar{z}_{k n, p n}^{t+1}\right|^{2}=0 \\
\lim _{\psi_{k n, p n} \rightarrow \infty}\left|z_{k n, p n}-\bar{z}_{k n, p n}^{t+1}\right|^{2}=\left|\bar{z}_{k n, p n}^{t+1}\right|^{2}
\end{gathered}
$$

This implies choosing the minimum value for $\psi_{k n, p n}$ minimizes the lagrangian. Hence we can choose $\psi_{k n, p n}=-1+$ $\frac{\left|\bar{z}_{k n, p n}^{t+1}\right|}{\sqrt{\delta}}$ when $\left|\bar{z}_{k n, p n}^{t+1}\right|^{2} \geq \delta$ or $\psi_{k n, p n}=0$ otherwise. Note that $\psi_{k n, p n}=0$ means $\left|\bar{z}_{k n, p n}^{t+1}\right|^{2} \leq \delta$ and $z_{k n, p n}=\bar{z}_{k n, p n}^{t+1}$ and which is the first case of KKT condition 4. or $\psi_{k n, p n}=$ $-1+\frac{\left|z_{k n, p n}^{t+1}\right|}{\sqrt{\delta}}$ implies $z_{k n, p n}^{t+1}=\frac{\bar{z}_{k n, p n}^{t+1} \sqrt{\delta}}{\left|z_{k n, p n}^{t+1}\right|}$ and $\left|z_{k n, p n}^{t+1}\right|^{2}=\delta$ which becomes the second case of KKT condition 4. Finally we can write

$$
\psi_{k n, p n}=\max \left\{0,-1+\frac{\left|\bar{z}_{k n, p n}^{t+1}\right|}{\sqrt{\delta}}\right\}
$$

which completes the proof.

\section{APPENDIX B \\ Proof of THEOREM 1}

Proof: First to prove

$$
\zeta_{\rho}\left(\mathbf{s}^{t+1}, \xi^{t+1}, \mathbf{Z}^{t+1}, \mu^{t}\right)-\zeta_{\rho}\left(\mathbf{s}^{t}, \xi^{t}, \mathbf{Z}^{t}, \mu^{t}\right) \leq 0
$$

we use the fact that

$$
\begin{gathered}
\zeta_{\rho}\left(\mathbf{s}^{t+1}, \xi^{t+1}, \mathbf{Z}^{t+1}, \mu^{t}\right)-\zeta_{\rho}\left(\mathbf{s}^{t}, \xi^{t}, \mathbf{Z}^{t}, \mu^{t}\right) \equiv \\
\zeta_{\rho}\left(\mathbf{s}^{t+1}, \xi^{t+1}, \mathbf{Z}^{t}, \mu^{t}\right)-\zeta_{\rho}\left(\mathbf{s}^{t}, \xi^{t}, \mathbf{Z}^{t}, \mu^{t}\right)+ \\
\zeta_{\rho}\left(\mathbf{s}^{t+1}, \xi^{t+1}, \mathbf{Z}^{t+1}, \mu^{t}\right)-\zeta_{\rho}\left(\mathbf{s}^{t+1}, \xi^{t+1}, \mathbf{Z}^{t}, \mu^{t}\right)
\end{gathered}
$$

We can see that

$$
\begin{gathered}
\zeta_{\rho}\left(\mathbf{s}^{t+1}, \xi^{t+1}, \mathbf{Z}^{t}, \mu^{t}\right)-\zeta_{\rho}\left(\mathbf{s}^{t}, \xi^{t}, \mathbf{Z}^{t}, \mu^{t}\right) \equiv \\
\iota_{\gamma}\left(\overline{\mathbf{s}}^{t+1}, \mathbf{s}^{t}, \xi^{t}\right)-\iota_{\gamma}\left(\overline{\mathbf{s}}^{t}, \mathbf{s}^{t}, \xi^{t}\right)+
\end{gathered}
$$




$$
\begin{gathered}
\iota_{\gamma}\left(\overline{\mathbf{s}}^{t+1}, \mathbf{s}^{t+1}, \xi^{t}\right)-\iota_{\gamma}\left(\overline{\mathbf{s}}^{t+1}, \mathbf{s}^{t}, \xi^{t}\right)+ \\
\iota_{\gamma}\left(\overline{\mathbf{s}}^{t+1}, \mathbf{s}^{t+1}, \xi^{t+1}\right)-\iota_{\gamma}\left(\overline{\mathbf{s}}^{t+1}, \mathbf{s}^{t+1}, \xi^{t}\right)
\end{gathered}
$$

Using eq(25) $\overline{\mathbf{s}}$ is the minimizer of $\iota_{\gamma}(\overline{\mathbf{s}}, \mathbf{s}, \xi)$, which implies

$$
\iota_{\gamma}\left(\overline{\mathbf{s}}^{t+1}, \mathbf{s}^{t}, \xi^{t}\right)-\iota_{\gamma}\left(\overline{\mathbf{s}}^{t}, \mathbf{s}^{t}, \xi^{t}\right) \leq 0
$$

by definition. Next,

$$
\begin{gathered}
\iota_{\gamma}\left(\overline{\mathbf{s}}^{t+1}, \mathbf{s}^{t+1}, \xi^{t}\right)-\iota_{\gamma}\left(\overline{\mathbf{s}}^{t+1}, \mathbf{s}^{t}, \xi^{t}\right) \\
=\frac{1}{2 \gamma}\left\|\xi^{t} \mathbf{s}^{t+1}-\overline{\mathbf{s}}^{t+1}\right\|^{2}-\frac{1}{2 \gamma}\left\|\xi^{t} \mathbf{s}^{t}-\overline{\mathbf{s}}^{t+1}\right\|^{2} \\
=\frac{1}{2 \gamma} \sum_{\bar{n}}^{M N}\left|\xi^{t} e^{\angle s_{\bar{n}}^{t+1}}-\right| \bar{s}_{\bar{n}}^{t+1}\left|e^{\angle s_{\bar{n}}^{t+1}}\right|^{2} \\
-\frac{1}{2 \gamma} \sum_{\bar{n}}^{M N}\left|\xi^{t} e^{\angle s_{\bar{n}}^{t}}-\right| \bar{s}_{\bar{n}}^{t+1}\left|e^{\angle s_{\bar{n}}^{t+1}}\right|^{2} \\
\leq \frac{1}{2 \gamma} \sum_{\bar{n}}^{M N}\left|\xi^{t}-\right| \bar{s}_{\bar{n}}^{t+1}||^{2}-\frac{1}{2 \gamma} \sum_{\bar{n}}^{M N}\left|\xi^{t}-\right| \bar{s}_{\bar{n}}^{t+1}||^{2} \\
\text { using the fact } \angle s_{\bar{n}}^{t+1}=\angle \bar{s}_{\bar{n}}^{t+1} \text { and } \\
\left|\xi^{t}\right| e^{\angle s_{\bar{n}}^{t}}-\left.\left|\bar{s}_{\bar{n}}^{t+1}\right| e^{\angle s_{\bar{n}}^{t+1}}\right|^{2} \geq\left|\xi^{t}-\right| \bar{s}_{\bar{n}}^{t+1}||^{2} \\
\Rightarrow \iota_{\gamma}\left(\overline{\mathbf{s}}^{t+1}, \mathbf{s}^{t+1}, \xi^{t}\right)-\iota_{\gamma}\left(\overline{\mathbf{s}}^{t+1}, \mathbf{s}^{t}, \xi^{t}\right) \leq 0
\end{gathered}
$$

Following

$$
\begin{gathered}
\iota_{\gamma}\left(\overline{\mathbf{s}}^{t+1}, \mathbf{s}^{t+1}, \xi^{t+1}\right)-\iota_{\gamma}\left(\overline{\mathbf{s}}^{t+1}, \mathbf{s}^{t+1}, \xi^{t}\right) \\
\frac{1}{2 \gamma}\left\|\xi^{t+1} \frac{1}{\sqrt{M N}} e^{<\overline{\mathbf{s}}^{t+1}}-\overline{\mathbf{s}}^{t+1}\right\|^{2}+\frac{\beta}{2}\left\|\xi^{t+1} \overline{\mathbf{y}}^{t+1}-\overline{\mathbf{s}}^{t+1}\right\|^{2} \\
-\frac{1}{2 \gamma}\left\|\xi^{t} \frac{1}{\sqrt{M N}} e^{<\overline{\mathbf{s}}^{t+1}}-\overline{\mathbf{s}}^{t+1}\right\|^{2}+\frac{\beta}{2}\left\|\xi^{t} \overline{\mathbf{y}}^{t+1}-\overline{\mathbf{s}}^{t+1}\right\|^{2}
\end{gathered}
$$

By expanding and applying eq.(33) we can get

$$
\begin{gathered}
\frac{1}{2 \gamma}\left\|\xi^{t+1} \frac{1}{\sqrt{M N}} e^{\angle \overline{\mathbf{s}}^{t+1}}-\overline{\mathbf{s}}^{t+1}\right\|^{2}+\frac{\beta}{2}\left\|\xi^{t} \overline{\mathbf{y}}^{t+1}-\overline{\mathbf{s}}^{t+1}\right\|^{2} \\
=-\frac{1}{2} \xi^{t+1}\left(\frac{1}{\gamma}+\beta\|\mathbf{y}\|^{2}\right) \leq 0
\end{gathered}
$$

Noting that

$$
\frac{1}{2 \gamma}\left\|\xi^{t} \frac{1}{\sqrt{M N}} e^{\angle \overline{\mathbf{s}}^{t+1}}-\overline{\mathbf{s}}^{t+1}\right\|^{2}+\frac{\beta}{2}\left\|\xi^{t} \overline{\mathbf{y}}^{t+1}-\overline{\mathbf{s}}^{t+1}\right\|^{2} \geq 0
$$

we can reach at the fact that

$$
\iota_{\gamma}\left(\overline{\mathbf{s}}^{t+1}, \mathbf{s}^{t+1}, \xi^{t+1}\right)-\iota_{\gamma}\left(\overline{\mathbf{s}}^{t+1}, \mathbf{s}^{t+1}, \xi^{t}\right) \leq 0
$$

Using eq (42),(43)and (44) we can deduce that

$$
\zeta_{\rho}\left(\mathbf{s}^{t+1}, \xi^{t+1}, \mathbf{Z}^{t}, \mu^{t}\right)-\zeta_{\rho}\left(\mathbf{s}^{t}, \xi^{t}, \mathbf{Z}^{t}, \mu^{t}\right) \leq 0
$$

Next,

$$
\zeta_{\rho}\left(\mathbf{s}^{t+1}, \xi^{t+1}, \mathbf{Z}^{t+1}, \mu^{t}\right)-\zeta_{\rho}\left(\mathbf{s}^{t+1}, \xi^{t+1}, \mathbf{Z}^{t}, \mu^{t}\right)
$$

$$
\begin{aligned}
& =\frac{\rho}{2} \sum_{p n=1}^{P n} \sum_{k n=1}^{K n}\left|z_{k n, p n}^{t+1}-\xi^{t+1} \widetilde{a}_{k n, p n}^{H} \widetilde{F}_{p n} s^{t+1}+\frac{\mu_{k n, p n}^{t}}{\rho}\right|^{2} \\
& -\frac{\rho}{2} \sum_{p n=1}^{P n} \sum_{k n=1}^{K n}\left|z_{k n, p n}^{t}-\xi^{t+1} \widetilde{a}_{k n, p n}^{H} \widetilde{F}_{p n} s^{t+1}+\frac{\mu_{k n, p n}^{t}}{\rho}\right|^{2} \\
& =\frac{\rho}{2} \sum_{p n=1}^{P n} \sum_{k n=1}^{K n}\left|z_{k n, p n}^{t+1}-\bar{z}_{k n, p n}^{t+1}\right|^{2}-\left|z_{k n, p n}^{t}-\bar{z}_{k n, p n}^{t+1}\right|^{2}, \\
& =\frac{\rho}{2} \sum_{p n=1}^{P n} \sum_{k n=1}^{K n}-\left|z_{k n, p n}^{t}-z_{k n, p n}^{t+1}\right|^{2}+2 \psi_{k n, p n}^{t+1} \Re\left(\left(z_{k n, p n}^{t}\right)^{H} z_{k n, p n}^{t+1}\right) \\
& -2 \psi_{k n, p n}^{t+1}\left|z_{k n, p n}^{t+1}\right|^{2},
\end{aligned}
$$

applying $\bar{z}_{k n, p n}^{t+1}=\xi^{t+1} \widetilde{a}_{k n, p n}^{H} \widetilde{F}_{p n} s^{t+1}+\frac{\mu_{k n, p n}^{t}}{\rho}$ using Lemma 3.

Then applying Lemma 3 we can have .

$$
\begin{gathered}
\frac{\rho}{2} \sum_{p n=1}^{P n} \sum_{k n=1}^{K n}-\left|z_{k n, p n}^{t}-z_{k n, p n}^{t+1}\right|^{2}+2 \psi_{k n, p n}^{t+1} \Re\left(\left(z_{k n, p n}^{t}\right)^{H} z_{k n, p n}^{t+1}\right) \\
-2 \psi_{k n, p n}^{t+1}\left|z_{k n, p n}^{t+1}\right|^{2} \leq \frac{\rho}{2} \sum_{p n=1}^{P n} \sum_{k n=1}^{K n}-\mid z_{k n, p n}^{t}- \\
\left.z_{k n, p n}^{t+1}\right|^{2}+2 \psi_{k n, p n}^{t+1}\left|z_{k n, p n}^{t}\right|\left|z_{k n, p n}^{t+1}\right|-2 \psi_{k n, p n}^{t+1}\left|z_{k n, p n}^{t+1}\right|^{2}
\end{gathered}
$$

Note that $0 \leq\left|z_{k n, p n}^{t}\right| \leq \sqrt{\delta} 0 \leq\left|z_{k n, p n}^{t+1}\right| \leq \sqrt{\delta}$ and $\psi_{k n, p n}^{t+1} \geq 0$ we have two cases that we need to consider for $k n=1 \ldots K n, p n=1 \ldots P n$

case1: $\psi_{k n, p n}^{t+1}=0$

$$
\begin{aligned}
& \frac{\rho}{2}\left(-\left|z_{k n, p n}^{t}-z_{k n, p n}^{t+1}\right|^{2}+2 \psi_{k n, p n}^{t+1}\left|z_{k n, p n}^{t}\right|\left|z_{k n, p n}^{t+1}\right|\right. \\
& \left.-2 \psi_{k n, p n}^{t+1}\left|z_{k n, p n}^{t+1}\right|^{2}\right) \\
& =-\left|z_{k n, p n}^{t}-z_{k n, p n}^{t+1}\right|^{2} \leq 0
\end{aligned}
$$

case2: $\psi_{k n, p n}^{t+1}>0, \Rightarrow\left|\bar{z}_{k n, p n}^{t+1}\right|>\left|z_{k n, p n}^{t+1}\right|=\sqrt{\delta}$,

$\left|z_{k n, p n}^{t}\right| \leq\left|z_{k n, p n}^{t+1}\right|$

If $\left|z_{k n, p n}^{t}\right|=\left|z_{k n, p n}^{t+1}\right|$

$$
\begin{aligned}
& \text { Then, } \frac{\rho}{2}\left(-\left|z_{k n, p n}^{t}-z_{k n, p n}^{t+1}\right|^{2}\right. \\
& \left.+2 \psi_{k n, p n}^{t+1}\left|z_{k n, p n}^{t}\right|\left|z_{k n, p n}^{t+1}\right|-2 \psi_{k n, p n}^{t+1}\left|z_{k n, p n}^{t+1}\right|^{2}\right) \\
& =-\left|\sqrt{\delta} e^{j \angle z_{k n, p n}^{t}}-e^{j \angle z_{k n, p n}^{t}}\right|^{2} \leq 0
\end{aligned}
$$

Else If $\left|z_{k n, p n}^{t}\right|<\left|z_{k n, p n}^{t+1}\right|$

Then, $2 \psi_{k n, p n}^{t+1}\left|z_{k n, p n}^{t}\right|\left|z_{k n, p n}^{t+1}\right| \leq 2 \psi_{k n, p n}^{t+1}\left|z_{k n, p n}^{t+1}\right|^{2}$, and $\frac{\rho}{2}\left(-\left|z_{k n, p n}^{t}-z_{k n, p n}^{t+1}\right|^{2}\right.$

$$
\left.+2 \psi_{k n, p n}^{t+1}\left|z_{k n, p n}^{t}\right|\left|z_{k n, p n}^{t+1}\right|-2 \psi_{k n, p n}^{t+1}\left|z_{k n, p n}^{t+1}\right|^{2}\right) \leq 0
$$

So,

$$
\frac{\rho}{2} \sum_{p n=1}^{P n} \sum_{k n=1}^{K n}-\mid z_{k n, p n}^{t}-
$$

$$
\left.z_{k n, p n}^{t+1}\right|^{2}+2 \psi_{k n, p n}^{t+1}\left|z_{k n, p n}^{t}\right|\left|z_{k n, p n}^{t+1}\right|-2 \psi_{k n, p n}^{t+1}\left|z_{k n, p n}^{t+1}\right|^{2} \leq 0
$$


$\Rightarrow \zeta_{\rho}\left(\mathbf{s}^{t+1}, \xi^{t+1}, \mathbf{Z}^{t+1}, \mu^{t}\right)-\zeta_{\rho}\left(\mathbf{s}^{t+1}, \xi^{t+1}, \mathbf{Z}^{t}, \mu^{t}\right) \leq 0$

Finally show that $\zeta_{\rho}\left(\mathbf{s}^{t+1}, \xi^{t+1}, \mathbf{Z}^{t+1}, \mu^{t+1}\right) \quad-$ $\zeta_{\rho}\left(\mathbf{s}^{t+1}, \xi^{t+1}, \mathbf{Z}^{t+1}, \mu^{t}\right)$ is bounded and converges to a limit point to conclude the convergence of the proposed solution. We can see that

$$
\begin{aligned}
& \zeta_{\rho}\left(\mathbf{s}^{t+1}, \xi^{t+1}, \mathbf{Z}^{t+1}, \mu^{t+1}\right)-\zeta_{\rho}\left(\mathbf{s}^{t+1}, \xi^{t+1}, \mathbf{Z}^{t+1}, \mu^{t}\right) \\
& =\frac{\rho}{2} \sum_{p n=1}^{P n} \sum_{k n=1}^{K n}\left(\left|z_{k n, p n}^{t+1}-\xi^{t+1} \widetilde{a}_{k n, p n}^{H} \widetilde{F}_{p n} s^{t+1}+\frac{\mu_{k n, p n}^{t+1}}{\rho}\right|^{2}\right. \\
& \left.-\left|\frac{\mu_{k n, p n}^{t+1}}{\rho}\right|^{2}\right) \\
& -\frac{\rho}{2} \sum_{p n=1}^{P n} \sum_{k n=1}^{K n}\left|z_{k n, p n}^{t+1}-\xi^{t+1} \widetilde{a}_{k n, p n}^{H} \widetilde{F}_{p n} s^{t+1}+\frac{\mu_{k n, p n}^{t}}{\rho}\right|^{2} \\
& \left.-\left|\frac{\mu_{k n, p n}^{t}}{\rho}\right|^{2}\right) \\
& =\frac{\rho}{2} \sum_{p n=1}^{P n} \sum_{k n=1}^{K n}\left(\left|\frac{\mu_{k n, p n}^{t+1}}{\rho}+\frac{\mu_{k n, p n}^{t+1}}{\rho}-\frac{\mu_{k n, p n}^{t}}{\rho}\right|^{2}\left|\frac{\mu_{k n, p n}^{t+1}}{\rho}\right|^{2}\right) \\
& \left.-\left|\frac{\mu_{k n, p n}^{t+1}}{\rho}\right|^{2}+\left|\frac{\mu_{k n, p n}^{t}}{\rho}\right|^{2}\right) \\
& =\frac{\rho}{2} \sum_{p n=1}^{P n} \sum_{k n=1}^{K n} 2\left|\frac{\mu_{k n, p n}^{t+1}}{\rho}-\frac{\mu_{k n, p n}^{t}}{\rho}\right|^{2} \\
& =\frac{1}{\rho} \sum_{p n=1}^{P n} \sum_{k n=1}^{K n}\left|\mu_{k n, p n}^{t+1}-\mu_{k n, p n}^{t}\right|^{2} \\
& =\rho \sum_{p n=1}^{P n} \sum_{k n=1}^{K n}\left|z_{k n, p n}^{t+1}-\xi^{t+1} \widetilde{a}_{k n, p n}^{H} \widetilde{F}_{p n} s^{t+1}\right|^{2}
\end{aligned}
$$

Taking the fact that $z_{k n, p n}^{t+1}, \xi^{t+1}, \mathbf{s}^{t+1}$ are the minimizers of the augmented lagrangian and

$$
\begin{gathered}
\lim _{t \rightarrow \infty}\left\{\mathbf{s}^{t+1}, \xi^{t+1},\left\{z_{k n, p n}^{t+1}\right\}_{(k n, p n)}\right\} \\
\rightarrow\left\{\mathbf{s}^{*}, \xi^{*},\left\{z_{k n, p n}^{*}\right\}_{(k n, p n)}\right\}, \\
(k n, p n) \in \bar{\Omega}
\end{gathered}
$$

Then,

$$
\left|\frac{\mu_{k n, p n}^{t+1}}{\rho}-\frac{\mu_{k n, p n}^{t}}{\rho}\right|^{2}=\left|z_{k n, p n}^{*}-\xi^{*} \widetilde{a}_{k n, p n}^{H} \widetilde{F}_{p n} s^{*}\right|^{2}
$$

is the limit point and Under the assumption

$$
\begin{gathered}
\lim _{t \rightarrow \infty}\left\|\mu^{t+1}-\mu^{t}\right\|^{2} \rightarrow 0 \\
\zeta_{\rho}\left(\mathbf{s}^{t+1}, \xi^{t+1}, \mathbf{Z}^{t+1}, \mu^{t+1}\right)-\zeta_{\rho}\left(\mathbf{s}^{t+1}, \xi^{t+1}, \mathbf{Z}^{t+1}, \mu^{t}\right) \\
=\rho \sum_{p n=1}^{P n} \sum_{k n=1}^{K n}\left|z_{k n, p n}^{*}-\xi^{*} \widetilde{a}_{k n, p n}^{H} \widetilde{F}_{p n} s^{*}\right|^{2}
\end{gathered}
$$

is bounded and has a converging point.

From eq(45), (46), (47), and (48), and taking the fact that (29) is convergent and hence (28) [35] we know that $0 \in \partial_{s} \zeta_{\rho}(\mathbf{s}, \xi, \mathbf{Z}, \mu)$ and $0 \in \partial_{\xi} \zeta_{\rho}(\mathbf{s}, \xi, \mathbf{Z}, \mu)$ and the error in the dual variable is bounded and has a limit point. Therefore we can deduce that $\left\{\mathbf{s}^{*}, \xi^{*},\left\{z_{k n, p n}^{*}\right\}_{(k n, p n) \in \bar{\Omega}},\left\{\mu_{k n, p n}^{*}\right\}_{(k n, p n) \in \bar{\Omega}}\right\}$ is a limit point and Algorithm1 is converging.

\section{REFERENCES}

[1] S. D. Blunt, M. Cook, J. Jakabosky, J. De Graaf, and E. Perrins, "Polyphase-coded fm (pcfm) radar waveforms, part i: implementation," IEEE Transactions on Aerospace and Electronic Systems, vol. 50, no. 3, pp. 2218-2229, 2014.

[2] P. M. McCormick, S. D. Blunt, and J. G. Metcalf, "Joint spectrum/beampattern design of wideband fm mimo radar emissions," in 2016 IEEE Radar Conference (RadarConf), pp. 1-6, IEEE, 2016.

[3] D. Zhao, Y. Wei, and Y. Liu, "Pcfm radar waveform design with spectral and correlation considerations," IEEE Transactions on Aerospace and Electronic Systems, vol. 53, no. 6, pp. 2885-2898, 2017.

[4] T. Jiang and Y. Wu, "An overview: Peak-to-average power ratio reduction techniques for ofdm signals," IEEE Transactions on broadcasting, vol. 54, no. 2, pp. 257-268, 2008.

[5] H. Griffiths, L. Cohen, S. Watts, E. Mokole, C. Baker, M. Wicks, and S. Blunt, "Radar spectrum engineering and management: Technical and regulatory issues," Proceedings of the IEEE, vol. 103, no. 1, pp. 85-102, 2014.

[6] H. Griffiths, S. Blunt, L. Cohen, and L. Savy, "Challenge problems in spectrum engineering and waveform diversity," in 2013 IEEE Radar Conference (RadarCon13), pp. 1-5, IEEE, 2013.

[7] D. Zhao, Y. Wei, and Y. Liu, "Over-sampled polyphase code design for physical implementation with spectral and correlation consideration," in 2016 IEEE Radar Conference (RadarConf), pp. 1-6, IEEE, 2016.

[8] S. D. Blunt and E. L. Mokole, "Overview of radar waveform diversity," IEEE Aerospace and Electronic Systems Magazine, vol. 31, no. 11, pp. 2 42, 2016.

[9] A. Aubry, A. De Maio, M. Piezzo, and A. Farina, "Radar waveform design in a spectrally crowded environment via nonconvex quadratic optimization," IEEE Transactions on Aerospace and Electronic Systems, vol. 50, no. 2, pp. 1138-1152, 2014.

[10] A. Aubry, A. De Maio, Y. Huang, M. Piezzo, and A. Farina, "A new radar waveform design algorithm with improved feasibility for spectral coexistence," IEEE Transactions on Aerospace and Electronic Systems, vol. 51, no. 2, pp. 1029-1038, 2015.

[11] G. Cui, X. Yu, G. Foglia, Y. Huang, and J. Li, "Quadratic optimization with similarity constraint for unimodular sequence synthesis," IEEE Transactions on Signal Processing, vol. 65, no. 18, pp. 4756-4769, 2017.

[12] P. M. McCormick, S. D. Blunt, and J. G. Metcalf, "Wideband mimo frequency-modulated emission design with space-frequency nulling," IEEE Journal of Selected Topics in Signal Processing, vol. 11, no. 2, pp. 363-378, 2016

[13] E. Raei, M. Alaee-Kerahroodi, and B. S. MR, "Beampattern shaping for coexistence of cognitive mimo radar and mimo communications," in 2020 IEEE 11th Sensor Array and Multichannel Signal Processing Workshop (SAM), pp. 1-5, IEEE, 2020.

[14] N. Levanon and E. Mozeson, Radar signals. John Wiley \& Sons, 2004.

[15] G. San Antonio and D. R. Fuhrmann, "Beampattern synthesis for wideband mimo radar systems," in 1st IEEE International Workshop on Computational Advances in Multi-Sensor Adaptive Processing, 2005., pp. 105-108, IEEE, 2005.

[16] H. L. Van Trees, Optimum array processing: Part IV of detection, estimation, and modulation theory. John Wiley \& Sons, 2004.

[17] H. He, P. Stoica, and J. Li, "Wideband mimo systems: Signal design for transmit beampattern synthesis," IEEE transactions on Signal Processing, vol. 59, no. 2, pp. 618-628, 2010.

[18] Y. Tang, Y. D. Zhang, M. G. Amin, and W. Sheng, "Wideband multipleinput multiple-output radar waveform design with low peak-to-average ratio constraint," IET Radar, Sonar \& Navigation, vol. 10, no. 2, pp. 325 332, 2016.

[19] H. Liu, X. Wang, B. Jiu, J. Yan, M. Wu, and Z. Bao, "Wideband mimo radar waveform design for multiple target imaging," IEEE Sensors Journal, vol. 16, no. 23, pp. 8545-8556, 2016.

[20] O. Aldayel, V. Monga, and M. Rangaswamy, "Tractable transmit mimo beampattern design under a constant modulus constraint," IEEE Transactions on Signal Processing, vol. 65, no. 10, pp. 2588-2599, 2017.

[21] K. Alhujaili, V. Monga, and M. Rangaswamy, "Mimo radar beampattern design under joint constant modulus and orthogonality constraints," in 2018 52nd Asilomar Conference on Signals, Systems, and Computers, pp. 1899-1904, IEEE, 2018. 
[22] B. Kang, O. Aldayel, V. Monga, and M. Rangaswamy, "Spatio-spectral radar beampattern design for coexistence with wireless communication systems," IEEE Transactions on Aerospace and Electronic Systems, vol. 55, no. 2, pp. 644-657, 2018.

[23] X. Yu, G. Cui, T. Zhang, and L. Kong, "Constrained transmit beampattern design for colocated mimo radar," Signal Processing, vol. 144, pp. 145-154, 2018.

[24] X. Yu, G. Cui, J. Yang, and L. Kong, "Wideband mimo radar beampattern shaping with space-frequency nulling," Signal Processing, vol. 160, pp. 80-87, 2019.

[25] X. Yu, J. Yang, G. Cui, and L. Kong, "Constant modulus waveform design for wideband mimo radar beampattern," in 2019 IEEE Radar Conference (RadarConf), pp. 1-5, IEEE, 2019.

[26] X. Yu, G. Cui, J. Yang, L. Kong, and J. Li, "Wideband mimo radar waveform design," IEEE Transactions on Signal Processing, vol. 67, no. 13, pp. 3487-3501, 2019.

[27] W. Fan, J. Liang, G. Lu, X. Fan, and H. C. So, "Spectrally-agile waveform design for wideband mimo radar transmit beampattern synthesis via majorization-admm,' IEEE Transactions on Signal Processing, vol. 69, pp. 1563-1578, 2021.

[28] S. D. Blunt, J. Jakabosky, M. Cook, J. Stiles, S. Seguin, and E. Mokole, "Polyphase-coded fm (pcfm) radar waveforms, part ii: optimization," IEEE Transactions on Aerospace and Electronic Systems, vol. 50, no. 3, pp. 2230-2241, 2014.

[29] J. G. Proakis, Digital signal processing: principles algorithms and applications. Pearson Education India, 2001.

[30] A. V. Oppenheim, J. R. Buck, and R. W. Schafer, Discrete-time signal processing. Vol. 2. Upper Saddle River, NJ: Prentice Hall, 2001.

[31] T. Qiu, P. Babu, and D. P. Palomar, "Prime: Phase retrieval via majorization-minimization," IEEE Transactions on Signal Processing, vol. 64, no. 19, pp. 5174-5186, 2016.

[32] S. Boyd, N. Parikh, and E. Chu, Distributed optimization and statistical learning via the alternating direction method of multipliers. Now Publishers Inc, 2011.

[33] D. R. Jachowski and C. Rauscher, "Frequency-agile bandstop filter with tunable attenuation," in 2009 IEEE MTT-S International Microwave Symposium Digest, pp. 649-652, IEEE, 2009.

[34] F. H. Raab, P. Asbeck, S. Cripps, P. B. Kenington, Z. B. Popovic, N. Pothecary, J. F. Sevic, and N. O. Sokal, "Power amplifiers and transmitters for $\mathrm{rf}$ and microwave," IEEE transactions on Microwave Theory and Techniques, vol. 50, no. 3, pp. 814-826, 2002.

[35] K. Huang and N. D. Sidiropoulos, "Consensus-admm for general quadratically constrained quadratic programming," IEEE Transactions on Signal Processing, vol. 64, no. 20, pp. 5297-5310, 2016.

[36] Y. Wang, W. Yin, and J. Zeng, "Global convergence of admm in nonconvex nonsmooth optimization," Journal of Scientific Computing, vol. 78, no. 1, pp. 29-63, 2019.

[37] M. Hong, Z.-Q. Luo, and M. Razaviyayn, "Convergence analysis of alternating direction method of multipliers for a family of nonconvex problems," SIAM Journal on Optimization, vol. 26, no. 1, pp. 337-364, 2016. 